\title{
Three-Dimensional Linear Instability of Persistent Anomalous Large-Scale Flows
}

\author{
ANDREW W. ROBERTSON AND WERNER METZ \\ Meteorologisches Institut, Universität München, München, Federal Republic of Germany
}

(Manuscript received 29 November 1988, in final form 17 March 1989)

\begin{abstract}
The linear baroclinic instability of three-dimensional basic flows on the Northern Hemisphere is examined in terms of a simple two-level, quasi-geostrophic model. The basic flows considered comprise an observed sixwinter mean flow, as well as anomalous flows which represent episodes where large-scale persistent flow anomalies, such as the Pacific/North American (PNA), East Atlantic (EA), or North Atlantic Oscillation (NAO) patterns exhibit large amplitudes.

For the climatological basic state, the fastest-growing normal modes with periods of around 4 days consist of regionally confined, synoptic-scale, baroclinic wave trains. These are considered as cyclogenesis modes, characterizing the linear synoptic-scale eddy activity associated with a given basic flow. This eddy activity has a pronounced maximum over the Pacific, close to the position of the observed Pacific storm track, but the second maximum over the Atlantic, corresponding to the Atlantic storm track, is considerably underestimated. Nevertheless, comparing the structure of the cyclogenesis modes with that of the leading complex EOFs of the observed bandpass-filtered flow, a pattern correlation squared of up to 0.4 is obtained. Truncating the basic state to comprise only the ultralong waves (zonal wavenumber $m \leqslant 4$ ) results in rather little change in the cyclogenesis modes obtained.

Finally, the sensitivity of the cyclogenesis modes to the anomalous basic flows is investigated, using persistent anomaly patterns (PNA, EA, NAO) obtained from a rotated principal component analysis of the observed lowpass-filtered flow. The anomalous basic states are evaluated by adding or subtracting these patterns to/from the winter climatological mean flow. It turns out that the normal-mode wave trains are significantly deflected from their climatological positions, particularly in the EA and NAO cases. This model response is verified against composite maps of observed bandpass variance, obtained for episodes of strong PNA, EA or NAO anomalies respectively. It is found that, although the normal-mode wave trains are still relatively too weak over the Atlantic (compared to the Pacific), the structural differences in the observed bandpass eddy activity between positive and negative anomaly cases are captured quite well by the normal modes.
\end{abstract}

\section{Introduction}

The importance of the synoptic-scale eddies in maintaining the time-mean flow, and for the dynamics of atmospheric low-frequency variability, has been a focus of interest in recent years. In particular, it is becoming increasingly clear that eddy momentum fluxes associated with the synoptic-scale eddies play an important role in maintaining large-scale persistent anomalies such as blocks and cut-off lows (Illari and Marshall 1983; Hoskins et al. 1985; Shutts 1986). This makes the geographical distribution of cyclogenesis and the storm tracks, as well as its variability, important for the dynamics of atmospheric low-frequency flow. Indeed, Vautard and Legras (1988) have shown that persistent large-scale flow regimes may result from a nonlinear equilibration of the synoptic-scale eddies with the planetary-scale flow. Egger and Schilling $(1983,1984)$ and Metz (1987) have found that the

Corresponding author address: Dr. Andrew W. Robertson, Meteorologisches Institut, Universitat Munchen, Theresienstrasse 37, D-8000 Munchen 2, Federal Republic of Germany. low-frequency variance maxima of a planetary-scale barotropic model winter climatology compared quite well with atmospheric observations, when the model was forced with the vorticity flux convergences associated with the observed synoptic-scale eddies. They were able, in addition, to model the temporal variability of this "synoptic-scale forcing" stochastically. It is natural to wonder if it would not be possible to close such a model by parameterizing the synoptic-scale eddies in terms of the model's planetary waves.

The normal-mode approach to baroclinic instability of three-dimensionally varying flows, pioneered by Frederiksen (1978b), has been rather successful in explaining the basic features of regional cyclogenesis. Frederiksen has found that the most-unstable normal modes of a quasi-geostrophic two-level model are locally organized and reach their maximum amplitude somewhat downstream of the regions of maximum baroclinity. Since then, Frederiksen (1982) has considered the linear, zonally inhomogeneous baroclinic stability problem associated with a northern hemispheric winter climatological mean flow. The fastestgrowing mode is a cyclogenesis mode with its maximum amplitude in the regions of the observed storm 
tracks, although it is strong right across North America. The mode has a growth rate of $0.43 \mathrm{day}^{-1}$, a period of 3.3 days, a dominant zonal wavenumber 9 , tilts westward with height, and has a monopole structure which is elongated in the meridional direction, making its characteristics rather typical of observed synoptic-scale eddies. Pierrehumbert (1986) has shown, also using a two-level model, that such linear modes evolve along an idealized storm track, from being shallow and highly baroclinic, to becoming concentrated aloft and rather barotropic. Thus linear modes arising from a zonally asymmetric basic state possess many of the attributes of observed synoptic-scale eddies. Additionally, although the synoptic eddies interact strongly with the planetary waves which have a large low-frequency variability, the observed mean storm tracks appear to be given surprisingly well in terms of a linear stability analysis of the climatological stationary waves.

It is well known (Blackmon and White 1982) that filtering in time (at about 10 days), clearly separates the high-frequency, synoptic-scale eddies, from the lowfrequency planetary-scale flow, whose variability dominates the total atmospheric variability. This, together with the above results, raises the question as to whether the normal-mode approach could be used to parameterize the synoptic eddies in a low-order model, along the lines of Rheinhold and Pierrehumbert (1982), and, in addition, whether it could also be applied to investigate regional cyclogenesis on intraseasonal time scales.

The aim of this work is to investigate the sensitivity of the linear cyclogenesis modes to changes in the basic state. We first consider the effect of truncating the basic state to comprise only the largest planetary scales (zonal wavenumber $m \leqslant 4$ ), in order to examine the possibility of using a linear stability analysis in a low-order climate model as part of a parameterization method for the effects of the synoptic eddies on the large-scale flow. Second, we investigate the impact of anomalous basic flows on the linear cyclogenesis modes. Coherent shifts in the storm tracks associated with the presence of persistent large-scale anomalies have indeed been found by Dole (1986) in a composite analysis of observational data. In particular we consider anomalous basic states associated with situations where the largescale flow is significantly changed due to the presence of persistent anomalies such as the Pacific/North American (PNA) pattern, the East Atlantic pattern (EA), and the North Atlantic Oscillation (NAO).

We employ a simple two-level quasi-geostrophic framework for the stability analysis, which is described in section 2. In order to examine the properties of the stability analysis, we first consider (in section 3 ) a simple idealized basic state, made up of a zonally symmetric flow and simple local anomalies. In section 4, we apply the analysis to a Northern Hemisphere winter climatological flow and compare our results with those of Frederiksen (1982). Here we investigate the effect on the analysis of highly truncating the basic state, in- cluding Ekman pumping, as well as sensitivity to the: static stability parameter. In section 5, we apply the: linear stability analysis to anomalous low-frequency' flows, which are obtained by superimposing selected rotated real empirical orthogonal functions (EOFs) derived from low-pass filtered winter data, onto oul: climatology. A summary and conclusion follow in sec. tion 6 .

\section{The linear stability analysis}

\section{a. The model}

The model used is a quasi-geostrophic spectral twolevel model on the hemisphere, which corresponds to the B-model of Frederiksen (1978b). The barotropic and baroclinic modes of the streamfunction are defined, as usual, by

$$
\psi=\frac{1}{2}\left(\psi_{250 \mathrm{mb}}+\psi_{750 \mathrm{mb}}\right)
$$

and

$$
\tau=\frac{1}{2}\left(\psi_{250 \mathrm{mb}}-\psi_{750 \mathrm{mb}}\right)
$$

respectively. Using the appropriate form of the thermal wind equation (with $f=f_{0}$ ) the model equations are written:

$$
\begin{gathered}
\partial \nabla^{2} \psi / \partial t=-J\left(\psi, \nabla^{2} \psi\right)-J\left(\tau, \nabla^{2} \tau\right) \\
-\left(2 \Omega / a^{2}\right) \partial \psi / \partial \lambda+K_{B} \nabla^{2} \psi_{s}+F_{\psi} \\
\partial\left\{\left(\nabla^{2}-\Lambda^{-2}\right) \tau\right\} / \partial t=-J\left(\psi,\left(\nabla^{2}-\Lambda^{-2}\right) \tau\right) \\
-J\left(\tau, \nabla^{2} \psi\right)-\left(2 \Omega / a^{2}\right) \partial \tau / \partial \lambda-K_{B} \nabla^{2} \psi_{s}+F_{\tau}
\end{gathered}
$$

where

$$
\Lambda=\left(b_{1} c_{p} \frac{1}{2} \Delta \theta_{0} / f_{0}^{2}\right)^{1 / 2}
$$

and $b_{1}=0.124$ (Lorenz 1960), $a$ is the earth's radius, $\Omega$ its angular velocity, $f_{0}$ the Coriolis parameter at $45^{\prime \prime}$ latitude, $\lambda$ is longitude and $\mu=\sin$ (lat). The streamfunction at the ground surface, $\psi_{s}$, is taken as $\frac{1}{2} \psi_{750 \mathrm{~m}}$, $=\frac{1}{2}(\psi-\tau)$, and $K_{B}=(7 \text { days })^{-1}$ is the coefficient of surface friction. The internal deformation radius, $\Lambda$, is a function of the static stability alone, which is given by the mean shear in potential temperature between the upper and lower levels $\left(\Delta \theta_{0}\right)$. The effects of diabatic heating, topography, and dissipation are represented by $F_{\downarrow}$ and $F_{r}$.

The eigenvalue problem associated with this model is explicit, and thus straightforward. Although the: model is very simple, we do not expect the results to differ greatly from those of a multilevel model. Fred. eriksen (1979b) has found that the effect of increasing; the number of levels is primarily to modify the vertical structure of the eigenmodes, rather than to substantially' change their horizontal structure. 


\section{b. Method of solution}

Equations (1) and (2) are linearized about a timemean basic state $(\bar{\psi}, \bar{\tau})$, and the equations for the perturbations $\left(\psi^{\prime}=\psi-\bar{\psi}, \tau^{\prime}=\tau-\bar{\tau}\right)$ are then decomposed in terms of complex spherical harmonics by substituting for $\psi^{\prime}, \bar{\psi}, \tau^{\prime}$ and $\bar{\tau}$, e.g.,

$$
\psi^{\prime}(\lambda, \mu, t)=\sum_{m=-M}^{M} \sum_{n=|m|}^{N} \psi_{n}^{\prime m}(t) P_{n}^{m}(\mu) e^{i m \lambda}
$$

where $(N, M)$ is the spectral truncation, $\psi_{n}^{\prime-m}$ $=\left(\psi_{n}^{\prime m}\right)^{*}$ and ()$^{*}$ denotes the complex conjugate. Only hemispheric modes are retained, so that the second sum is over components with $(n-|m|)$ odd only, yielding $J=121$ complex coefficients for $M=N$ $=21$. The resulting equations are then multiplied through by $\left(P_{n}^{m} e^{i m \lambda}\right)^{*}$, and integrated over the hemisphere, using the orthogonality properties of spherical harmonics. This yields the following ordinary differential equations (dropping the indices $m, n$ ) for each spherical harmonic coefficient $j$, of the perturbation:

$$
\begin{aligned}
\frac{d}{d t} \gamma_{j} \psi_{j}^{\prime}= & -\sum_{k, l} i\left[\left(\bar{\psi}_{k} \psi_{l}^{\prime}+\psi_{k}^{\prime} \bar{\psi}_{l}\right)\right. \\
& \left.+\left(\bar{\tau}_{k} \tau_{l}^{\prime}+\tau_{k}^{\prime} \bar{\tau}_{l}\right)\right] L_{j k l}\left(\gamma_{l}-\gamma_{k}\right)-2 i m \psi_{j}^{\prime} \\
& -K_{B} \gamma_{j} \frac{1}{2}\left(\psi_{j}^{\prime}-\tau_{j}^{\prime}\right)
\end{aligned}
$$

$$
\begin{aligned}
& \frac{d}{d t}\left(\gamma_{j}-\Lambda^{-2}\right) \tau_{j}^{\prime} \\
& =-\sum_{k, l} i\left(\bar{\psi}_{k} \tau_{l}^{\prime}+\psi_{k}^{\prime} \bar{\tau}_{l}\right) L_{j k l}\left(\gamma_{l}-\gamma_{k}-\Lambda^{-2}\right) \\
& \quad-\sum_{k, l} i\left(\bar{\tau}_{k} \psi_{l}^{\prime}+\tau_{k}^{\prime} \bar{\psi}_{l}\right) L_{j k l}\left(\gamma_{l}-\gamma_{k}+\Lambda^{-2}\right) \\
& -2 i m \tau_{j}^{\prime}+K_{B} \gamma_{j} \frac{1}{2}\left(\psi_{j}^{\prime}-\tau_{j}^{\prime}\right)
\end{aligned}
$$

where $\gamma_{j}=-n_{j}\left(n_{j}+1\right)$, the $L_{j k l}$ are the interaction coefficients given by Elsaesser (1966), and we have nondimensionalized using length scale $a$, and time scale $\Omega^{-1}$. The double summations in the Jacobian terms are restricted to unique combinations of $k$ and lonly.

We split each spherical harmonic coefficient into its real and imaginary parts, e.g., $\psi_{j}^{\prime}=2^{-1 / 2}\left(\psi_{j c}^{\prime}-i \psi_{j s}^{\prime}\right)$, where subscripts $c$ and $s$ denote the cosine and sine components, respectively. Then splitting (4) and (5) into their real and imaginary parts and writing in matrix form yields

$$
d / d t\left(\mathbf{x}^{\prime}\right)=\mathbf{A} \cdot \mathbf{x}^{\prime}
$$

where $\mathbf{x}=\left(\psi_{1 c}, \cdots, \psi_{J c}, \psi_{1 s}, \cdots, \psi_{J s}, \tau_{1 c}, \cdots, \tau_{J c}\right.$, $\left.\tau_{1 s}, \cdots, \tau_{J s}\right)^{\mathrm{T}}$, and $\mathbf{A}=\mathbf{A}\left(\overline{\mathbf{x}}, \Lambda^{-2}, K_{B}\right)$ is the system matrix.

Solutions to the real matrix equation (6) have the normal mode form (dropping primes):

$$
\mathbf{x}(t)=\hat{\mathbf{x}} e^{-i \omega t},
$$

which, upon substitution into (6) yields the eigenproblem

$$
-i \omega \hat{\mathbf{x}}=\mathbf{A} \cdot \hat{\mathbf{x}}
$$

with the generally complex angular frequency $\omega=\omega_{r}$ $+i \omega_{i}$. The system matrix $\mathbf{A}$ is real, but, in general, not symmetric so the resulting eigenvectors $\mathbf{x}^{(k)}$ are complex (and in general not unitary) and generally come in complex conjugate pairs, as do the eigenvalues, $-i \omega^{(k)}$. With $\omega_{r}^{(k)} \neq 0$, we have propagating disturbances with angular frequency $\left|\omega_{r}{ }^{(k)}\right|$ and growth rate $\omega_{i}{ }^{(k)}$. The phase $\left(\omega_{r}{ }^{(k)} t\right)$, and the amplitude $\exp \left(\omega_{i}{ }^{(k)} t\right)$, of the eigenmodes depends on $t$ and are thus arbitrary, and we consider the modal structure at $t=0$. The oscillatory part of the normal mode actually consists of two patterns one-quarter period apart. However, for the cyclogenesis-type modes considered in the following, the quadrature phase is very similar in structure to that at $t=0$, being simply shifted a quarter wavelength eastward in space for $\omega_{r}{ }^{(k)}>0$, indicating that the modes propagate eastward.

The matrix A has size $484 \times 484$ for a T21 truncation [i.e., $N=M=21$ in (3)], and the eigenproblem (8) is solved using a NAG library routine. The surface friction terms are set to zero unless stated otherwise.

\section{c. The basic states}

Six winters of Northern Hemisphere winter geopotential height data from the European Centre for Medium Range Weather Forecasts (ECMWF) for the period $1979 / 80$ to $1984 / 85$ were used to construct the basic states. Each winter consists of 110 days of 0000 UTC daily analyses starting on 20 November. The geostrophic streamfunction is derived from the geopotential height by solving the linear balance equation (Metz 1986). We take the streamfunction at $850 \mathrm{mb}$ to be representative of the model's lower layer, and that at $300 \mathrm{mb}$ of the upper layer, so as to include lowlevel baroclinity to some extent. In general (unless stated otherwise), we use a T21 truncation for both the basic state and the perturbation. Following Frederiksen (1982), we assume that the basic state is balanced by $F_{\psi}$ and $F_{r}$, and so consider it as a stationary solution of (1) and (2).

The static stability parameter is chosen such that the fastest growing modes are realistic. When the perturbation is truncated at $\mathrm{T} 21, \Delta \theta_{0}=30-36 \mathrm{~K}$, which corresponds to an internal deformation radius of 419-459 $\mathrm{km}$, is found to yield modes with growth rates, phase speeds, and structures which compare reasonably with observed extratropical cyclones. The required $\Delta \theta_{0}$ is truncation dependent: increasing the horizonatal resolution decreases the growth rates of the fastest growing modes with attendant changes in phase speed and modal structure, as also found by Frederiksen and Bell (1987). 

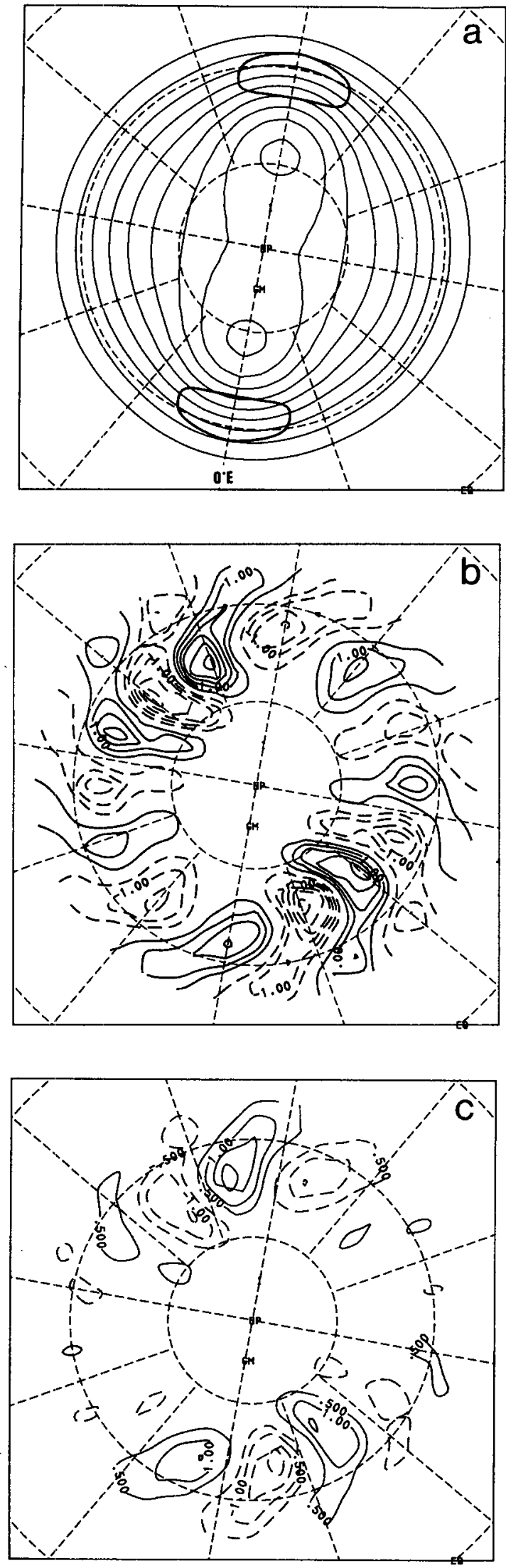

\section{Instability of idealized flows}

Figure $1 a$ illustrates an idealized basic state which has two identical jets situated $180^{\circ}$ apart. It consists of the zonally averaged six-winter climatology plus two identical nearly circular equivalent-barotropic streamfunction anomalies centered at $50^{\circ} \mathrm{N}$, computed according to the expression

$$
\begin{aligned}
\psi(\lambda, \phi)=B \exp \left\{-\left(x-x_{0}\right)^{2} / x_{*}^{2}\right\} & \\
& \times \exp \left\{-\left(y-y_{0}\right)^{2} / y_{*}^{2}\right\}
\end{aligned}
$$

where $B=30 \times 10^{6} \mathrm{~m}^{2} \mathrm{~s}^{-1}, \phi$ is latitude, and $x$ and $y$ are given by $x(\lambda, \phi)=\lambda \cos \phi$ and $y(\phi)=\tan \left\{\frac{1}{2}\left(90^{\circ}\right.\right.$ $-\phi)\}$. The anomaly is centered at $\left(x_{0}, y_{0}\right)$, where $x_{0}$ $=0$ and $y_{0}=y\left(50^{\circ}\right)$, and has Gaussian scales $x_{*}$ $=x\left(30^{\circ}, 45^{\circ}\right)$ and $y_{*}=y\left(45^{\circ}\right)-y\left(45^{\circ}+20^{\circ}\right)$. The amplitude of the $\tau$-anomaly is two-thirds that of the $\psi$-anomaly, whose amplitude is such as to produce a trough comparable with those of the winter climatology. The frictionless eigen-problem in section 2 is solved for this basic state with $\Delta \theta_{0}=36 \mathrm{~K}$; the perturbation is truncated at T21 and the basic state at T15.

The eigenvalues of the ten fastest-growing modes are given in Table 1. The modes have periods ranging from about 2-7 days, which coincides approximately with the bandpass filter window used by Blackmon (1976) to identify extratropical cyclones in observational data. The growth rates of the first five modes are rather bunched together, making it difficult to differentiate the modes by growth rate. The last column in Table 1 indicates the geographical distribution of model activity. Mode 1 , whose $\psi$ - and $\tau$-streamfunctions are illustrated in Figs. $1 \mathrm{~b}$ and $1 \mathrm{c}$ respectively, is typical of the "class A" modes, which are regional in nature and have short periods, but are not necessarily the fastest growing. Mode 1 reaches its maximum amplitude some $20^{\circ}-30^{\circ}$ downstream of the trough axes, and by virtue of the symmetrical nature of the basic state, has almost identical structure downstream of the two troughs. The mode has dominant zonal wavenumber 7 , maximum amplitude at $40^{\circ}-50^{\circ} \mathrm{N}$, and is monopole with a "beanlike" meridiónally elongated structure. The baroclinic streamfunction (Fig. 1c) indicates that the mode has a westward tilt with height. These characteristics of the fastest growing mode are in general agreement with Frederiksen (1978b), who considers the instability of various idealized zonally varying flows. In particular, Frederiksen (1978b) finds that the max-

FIG. 1. Linear stability analysis of zonally averaged winter climate, to which two identical negative streamfunction anomalies (see text) have been added at $0^{\circ}$ and $180^{\circ} \mathrm{W}$. (a) The basic state: contours of $\psi$ (thin contours) at intervals of $10 \times 10^{6} \mathrm{~m}^{2} \mathrm{~s}^{-1}$, and the $20 \mathrm{~m} \mathrm{~s}^{-1}$ isotach of the thermal wind $\left(\left|\mathbf{v}_{T}\right|\right)$ calculated from $\tau$ (thick contour). (b) and (c) The spatial structure of the fastest-growing mode, $\psi$-and $\tau$-streamfunction fields respectively (same contour interval). Magnitudes arbitrary, negative contours dashed, zero contour omitted. All maps are polar-stereographic projections as in Fig. 3. 
TABLE 1. The eigenvalues of the ten fastest-growing modes, obtained for the idealized basic state in Fig. 1a. The last column gives a subjective classification of the associated eigenmodes by structure. Class A-regional mode, class B-hemispheric mode, class C-regionally intensified hemispheric mode.

\begin{tabular}{ccccc}
\hline \hline $\begin{array}{c}\text { Mode } \\
k\end{array}$ & $\begin{array}{c}\text { Angular } \\
\text { frequency } \\
\left|\omega_{r}\right| \\
\left(\text { day }^{-1}\right)\end{array}$ & $\begin{array}{c}\text { Period, } \\
T \\
\text { (days) }\end{array}$ & $\begin{array}{c}\text { Growth rate, } \\
\omega_{i} \\
\text { (days }^{-1} \text { ) }\end{array}$ & Class \\
\hline 1 & 101 & 3.6 & 0.48 & A \\
2 & 77 & 4.7 & 0.46 & C \\
3 & 121 & 3.0 & 0.46 & A \\
4 & 93 & 3.9 & 0.45 & C \\
5 & 122 & 3.0 & 0.45 & A \\
6 & 77 & 4.7 & 0.43 & B \\
7 & 55 & 6.5 & 0.39 & B \\
8 & 66 & 5.5 & 0.35 & A \\
9 & 177 & 2.0 & 0.34 & C \\
10 & 51 & 7.1 & 0.34 & \\
\hline
\end{tabular}

imum development occurs slightly downstream of the positions where the "excess shear" is a maximum; the excess shear being defined as the difference between the actual vertical wind shear and that required by Phillips' (1954) criterion for incipient instability in the two-level model.

In contrast to the class $\mathrm{A}$ modes, there are others which have very little regional structure, and extend right around the hemisphere with almost no longitudinal modulation. These hemispheric modes are typical of those which result from a zonally averaged basic state; being a product of the instability of the zonally symmetric component of the basic state. A third class of modes has hemispheric extent, but are regionally intensified downstream of the jet maxima.

Pierrehumbert (1984) discusses the concept of "local" and "global" linear modes in the context of the stability characteristics of zonally varying flows. It is proposed that "global" modes, which are hemispheric in extent, result from "convective" instability, which depends essentially on the zonally averaged baroclinity and requires periodic recycling of energy around the hemisphere for growth. In this case the group velocity of a wave packet emanating from an initial disturbance exceeds the spatial spreading (or dispersion) of the packet, so that the mode dies out locally as it propagates away from the initial disturbance. On the other hand, "absolute" instability at any fixed point in longitude, may give rise to dynamically "local" modes. In this case, spatial spreading of the wave packet exceeds its group velocity, and if in addition, the peak of the mode dies out as it propagates out of the baroclinically unstable region, it may leave behind it an amplifying tail which manifests itself as a local mode. Pierrehumbert (1984) suggests that only such local modes are physically relevant to cyclogenesis, and that their growth rate depends on the maximum baroclinity in the domain and depends inversely on the strength of the zonal flow. The degree of localization is governed by the zonal baroclinity contrast.

The question arises as to whether our regional modes are indeed "local" in this sense, or if periodic recycling of energy is responsible for the growth. According to Pierrehumbert's (1984) analysis of a two-level model, a guide to whether local modes can occur is given approximately by the ratio of maximum to minimum wind shear around the hemisphere. Highly simplified analysis suggests that this ratio must be greater than 1.38 for local modes to occur in the two-level model. In our case (Fig. 1a) the ratio is about 1.64 at $35^{\circ} \mathrm{N}$, so that the regional modes may indeed be "local" in this sense. Evidence that absolute instability and its attendant local modes are not confined to the twolevel model is given by the multilevel stability analyses of Frederiksen (1979b), which show the same kind of horizontal structures as seen in the two-level model results.

Further experiments have been carried out with other idealized flows. When the basic state has only one trough instead of two, the results are little changed; there being now only a single modal storm track, although there tend to be fewer regional modes and more regionally intensified hemispheric modes. The main effect of adding a ridge downstream of an isolated trough (Fig. 2a) is to cause the modal storm track to split around the positive streamfunction anomaly, as illustrated by the fastest-growing mode in Fig. $2 \mathrm{~b}$. In the above experiments, the growth rate of the fastestgrowing mode was not found to change greatly. However, when the zonally symmetric part of the basic state is considered alone, the fastest growing mode has a somewhat higher growth rate of $0.56 \mathrm{day}^{-1}$, indicating that the equivalent barotropic anomalies have, in general, a stabilizing effect.

\section{Instability of a winter climatological flow}

In this section we consider the three-dimensional linear instability of the six-winter ECMWF climatology, illustrated in Fig. 3a. The trough over the western Pacific is stronger and larger scale than that over the east coast of North America, and much more baroclinic. Figure $3 b$ illustrates the standard deviation of the bandpass filtered $\psi$-streamfunction, where the filter (Blackmon and Lau 1980) retains oscillations with periods between approximately $2 \frac{1}{2}$ and 6 days. In contrast to the relative weakness of the Atlantic jet, the bandpass eddy activity over the northwest Atlantic is, if anything, stronger than that over the central Pacific. Both storm tracks lie downstream and slightly poleward of the climatological jets.

\section{a. Eigenmodes}

The frictionless eigenvalue problem in section 2 was solved for the above basic state, using $\Delta \theta_{0}=30 \mathrm{~K}$, and 

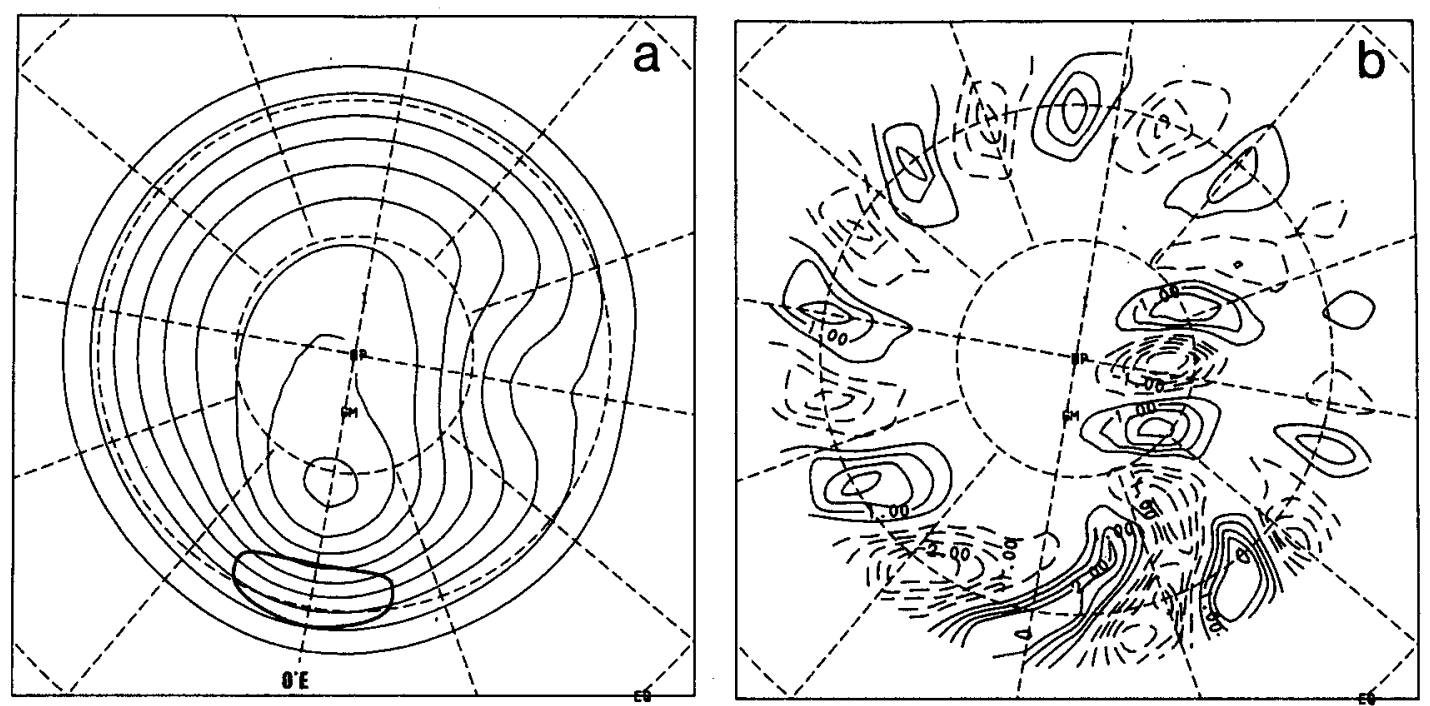

FIG. 2. As Fig. la and $1 \mathrm{~b}$, but for idealized basic state consisting of a trough at $0^{\circ} \mathrm{W}$ and a ridge of equal magnitude at $90^{\circ} \mathrm{E}$.

both basic state and perturbation truncated at $\mathrm{T} 21$. The complete spectrum of eigenvalues is shown in Fig. 4a, where for each complex-conjugate eigenvalue pair, only the member corresponding to nonnegative angular frequency is plotted. Because the effect of friction has been excluded, there are as many decaying as growing modes, and the eigenvalues are distributed rather symmetrically with respect to the zero growth rate axis. The majority of modes are concentrated about the origin of Fig. 4a; having small growth rates and large periods. Although the fastest-growing modes have similar growth rates, they have a broad range of frequencies.

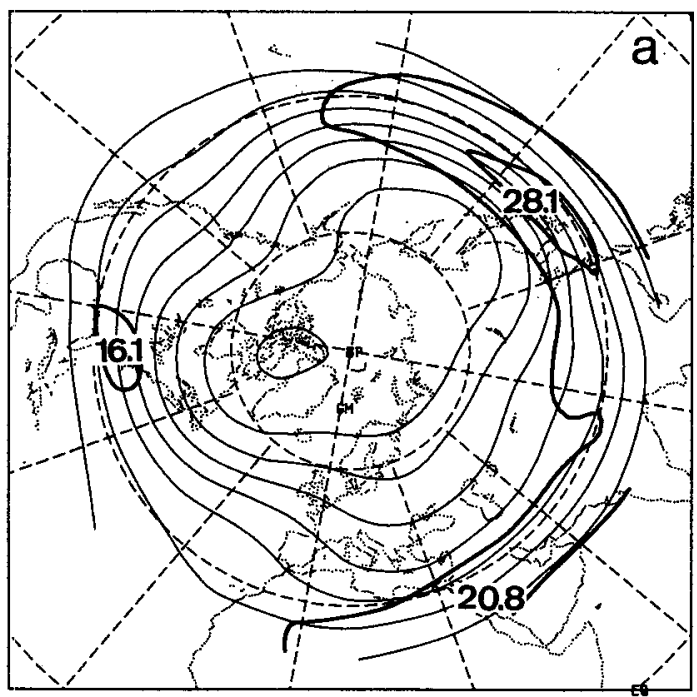

As in the previous section, a subjective classification of the spatial modal structures can be máde, as illu; trated in Fig. 4b, which shows the eigenvalues of the 20 fastest-growing modes (growth rate vs period); except mode 19 which has an infinite period. Here a primary distinction has been made between modes with storm-track like structures (synoptic scale, monopole zonal midlatitude wavetrains), and those with larger scale structures ("other modes" in Fig. 4b). The storm track modes may be subdivided according to their spatial scale and regionality, and in Fig. $4 \mathrm{~b}$ we distinguish between highly localized "Pacific" modes, "PacificAtlantic" modes which extend over both oceans, and

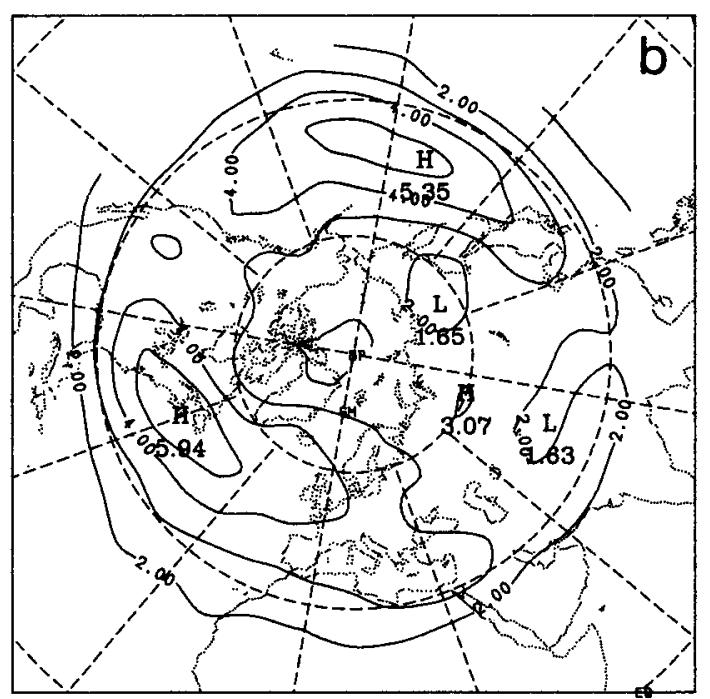

FIG. 3. ECMWF six-winter climatology (T21). (a) Time mean: contours of $\psi$ (thin contours) at intervals of $10 \times 10^{6} \mathrm{~m}^{2} \mathrm{~s}^{-1}$, and the 15 and $25 \mathrm{~m} \mathrm{~s}^{-1}$ isotachs of $\left|\mathbf{v}_{T}\right|$ (thick lines). Maxima of $\left|\mathbf{v}_{T}\right|\left(\mathrm{m} \mathrm{s}^{-1}\right)$ are also shown. (b) Standard deviation of bandpass filtered $\psi$; units $10^{6} \mathrm{~m}^{2} \mathrm{~s}^{-1}$. 

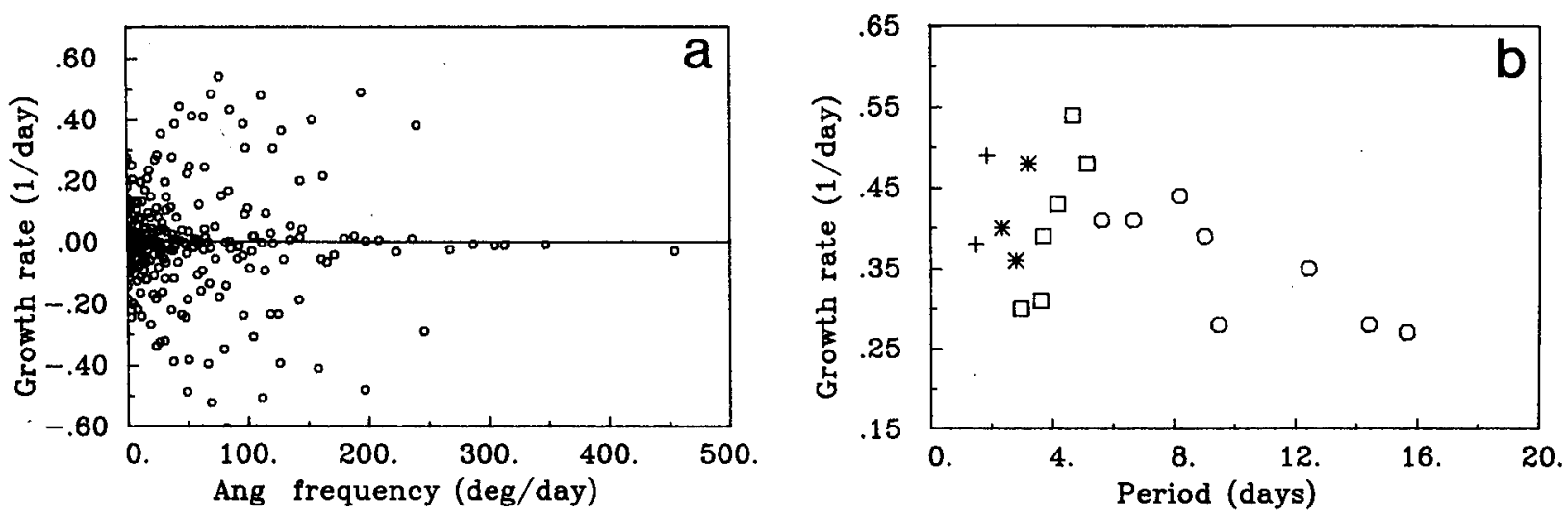

FIG. 4. Eigenvalues of the stability analysis with climatic basic state ( $\mathrm{T} 21, \Delta \theta_{0}=30 \mathrm{~K}$ ). (a) All eigenvalues, (b) first 20 by growth rate plotted against period (except mode 19 which has infinite period), together with a subjective classification of the associated eigenmode spatial structure, see text. Key to modal structure: $(+)$ "Pacific" modes, $(*)$ "Pacific-Atlantic" modes, $(\square)$ less regionally confined stormtrack modes, and $(O)$ other modes.

less longitudinally confined storm-track modes, which have quasi-hemispheric extent. As can be seen from Fig. $4 \mathrm{~b}$, the above classification is a strong function of modal period, and modes with periods less than about 4 days tend to be those with a meaningfully regional storm-track structure, which we refer to as cyclogenesis modes (Frederiksen 1982). It is found that eigenmodes with similar frequencies have structures which are quite consistent with one another.

Figure 5 illustrates the horizontal structures of the first six modes (classified by growth rate), with periods less than or equal to 4 days. For comparison, the two leading complex EOFs of the bandpass filtered ECMWF winter data, which both have periods near 4 days (and account for $12.2 \%$ and $11.2 \%$ of the variance respectively), are illustrated in Fig. 6. As is the case with the cyclogenesis-type eigenmodes, the quadrature phase structure of the complex EOFs closely resembles the one shown, being simply shifted a quarter wavelength in space. Both EOFs have similar structures over both Pacific and Atlantic, so that it is clear that they may not be interpreted individually as physical modes. Indeed, by evaluating the empirical standard errors of the EOF eigenvalues, it is found that these two EOFs are not distinct from one another (while being clearly distinct from the remaining EOFs), and must be considered together in order to represent the observed synoptic-scale eddies over the Pacific or Atlantic.

Modes 2 and 12 (Figs. 5a, e; modes ordered by growth rate, mode 1 being the fastest growing) have the shortest periods ( $T<2$ days), and are examples of the "Pacific" modes. They have intense local activity over the central Pacific in the region of the observed Pacific storm track. These modes apparently resemble the EOFs quite closely over the Pacific, both in location and structure, although they are slightly smaller in scale, and situated a few degrees further south. Modes 4, 9 and 13 (panels b, $c$ and $f$ in Fig. 5) are examples of "Pacific-Atlantic" modes, which are somewhat less lo- cally confined, having activity over the Atlantic and North America as well. These somewhat slower-oscillating modes have slightly larger spatial scales, more in agreement with the EOFs, but are not so locally confined over the Pacific or Atlantic. Mode 10 (Fig. $5 \mathrm{~d}$ ) is typical of those modes with periods between about 4 and 5 days, which are still less longitudinally confined, and have increasingly larger spatial scales.

It is noteworthy that there are no purely Atlantic modes among the cyclogenesis modes, and indeed very rarely does a mode have an Atlantic storm track which is more pronounced than that over the Pacific. However, the basic state (Fig. 3a) does indeed have much weaker baroclinity in the region of the Atlantic jet. A similar study by Frederiksen (1982), which also includes some nongeostrophic effects (the so-called " $P$ model"), is in general agreement with the above findings. However, the fastest-growing mode is found not only to be a cyclogenesis mode, but has maximum activity over the Atlantic. Although Frederiksen (1982) uses a somewhat higher spatial resolution for the perturbation, and a lower value of static stability $\left(\Delta \theta_{0}=23\right.$ $\mathrm{K}$ ), one wonders if these differences might be due to differences between the $B$-model (used here), and Frederiksen's $P$-model, which allows the Coriolis parameter to vary in the divergence term in the thermal vorticity equation, and in the thermal wind equation. Frederiksen (1978b) has compared the Phillips (1954) instability criterion for both models. He concludes that the $B$-model is increasingly more unstable than the $P$ model equatorward of about $45^{\circ}$, and slightly more stable than the $P$-model to the north. This probably explains why our Pacific modes are situated slightly too far south. Nevertheless it is puzzling, given the relatively weak baroclinity in the Atlantic jet and the small difference in latitude between the Pacific and Atlantic jet axes, why Frederiksen's Atlantic modal activity should be so strong. One possibility is that the strong baroclinic zone associated with the Pacific jet extends 

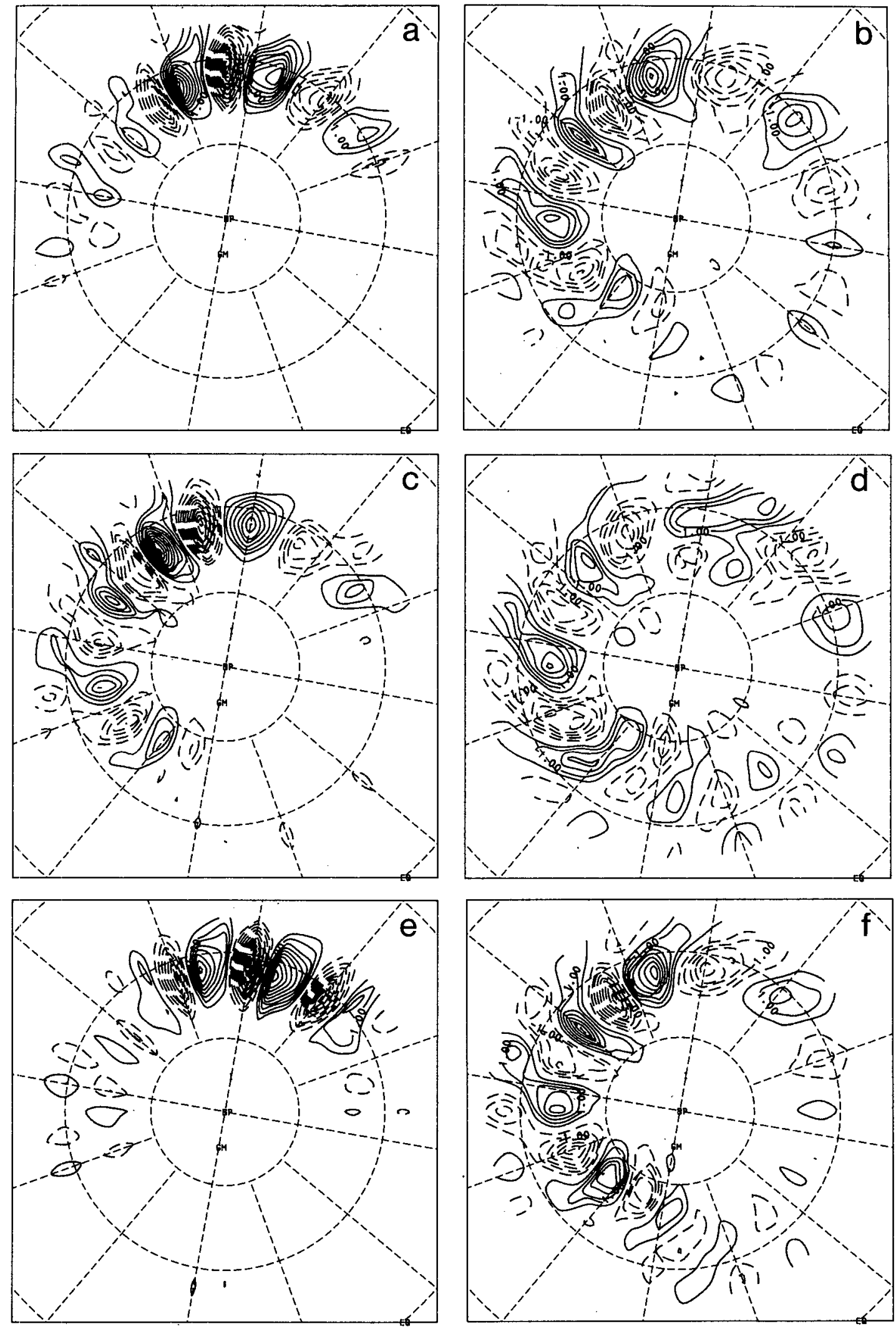

FIG. 5. The $\psi$-streamfunctions at time $t=0$ for the six fastest-growing modes with periods less than 4 days in Fig. 4 . Modes ordered by growth rate, modal period, in days, in brackets; (a) mode 2 (1.9), (b) mode 4 (3.2), (c) mode 9 (2.3), (d) mode 10 (3.7), (e) mode 12 $(1.5)$, (f) mode 13 (2.8). Otherwise as Fig. Ib. 

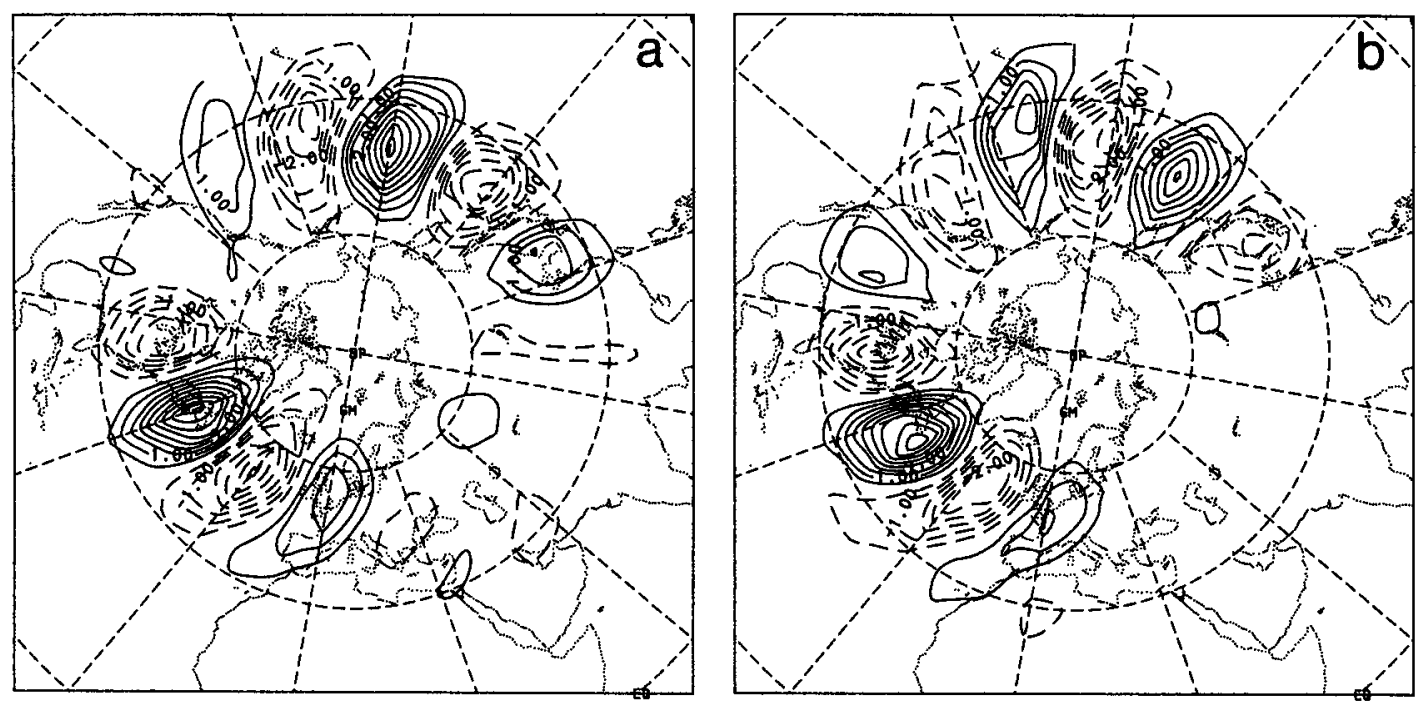

FIG. 6. The two leading complex EOFs of six-winter bandpass $\psi$ (T21) at arbitrary time. Contour interval $0.5 \times 10^{6} \mathrm{~m}^{2} \mathrm{~s}^{-1}$, negative dashed, zero contour omitted. Mode 1 (a) explains $12.2 \%$ of the variance and has a period of about 4.0 days. The figures for mode 2 (b) are $11.2 \%$ and 3.9 days, respectively.

appreciably southward of the jet axis, and may thus have an exaggerated influence on instability in the $B$ model.

\section{b. Pattern correlation}

In order to quantify the extent to which the spatial structures of fastest-growing modes resemble those of extratropical cyclones, the skill with which the first two complex bandpass EOFs (Fig. 6) taken together account for the spatial variance of each of the leading 20 eigenmodes has been computed. Because the EOFs are orthogonal by definition, the skill is simply given by where

$$
S^{(k)}=\left|C\left(\mathbf{x}^{(k)}, \mathrm{e}^{(l)}\right)\right|^{2}+\left|C\left(\mathbf{x}^{(k)}, \mathbf{e}^{(2)}\right)\right|^{2},
$$

$$
\begin{aligned}
C\left(\mathbf{x}^{(k)}, \mathbf{e}^{(l)}\right) & =\left(\mathbf{x}^{(k)} \cdot \mathbf{e}^{(l) *}\right) /\left(\left|\mathbf{x}^{(k)}\right|^{2}\left|\mathbf{e}^{(l)}\right|^{2}\right)^{1 / 2} \\
l & =1,2
\end{aligned}
$$

is the complex pattern correlation between the $\psi$ streamfunction vector of eigenmode $k, \mathbf{x}^{(k)}$, and that of EOF $l, \mathbf{e}^{(l)}$. By performing the pattern correlations in spectral space, the arbitrary phases of the EOFs and eigenmodes are of no concern. With 121 spatial degrees of freedom, a one-tailed test of the pattern correlation suggests skill scores of $>0.15$ to be statistically highly significant ( $99.9 \%$ level).

Figure 7 illustrates the skills $S^{(k)}$ for the 20 fastestgrowing modes (again mode 19 omitted), together with the subjective mode classification of Fig. $4 b$ and the mode index. The skill is clearly a function of model period, as was our subjective classification. However, the skill peaks at about 4 days, and the modes with the highest skills are those with quite extensive stormtracklike structures, whose spatial scale and latitude compare well with the EOFs. The very localized Pacific modes are too small scale, and are situated too far south to correlate well with the EOFs. Nevertheless, the skill scores generally support our selection by frequency of the cyclogenesis modes.

\section{c. Static stability parameter}

In order to investigate the effect of changing the static stability parameter, the above analysis was repeated with $\Delta \theta_{0}=24$ and $36 \mathrm{~K}$. Figure 8 illustrates the eigenvalues of the 20 fastest-growing modes, together with approximate values of $S^{(k)}$ for each mode for the three cases $\Delta \theta_{0}=24,30$ and $36 \mathrm{~K}$ (modes with periods $>20$ days have again been omitted). While the most obvious

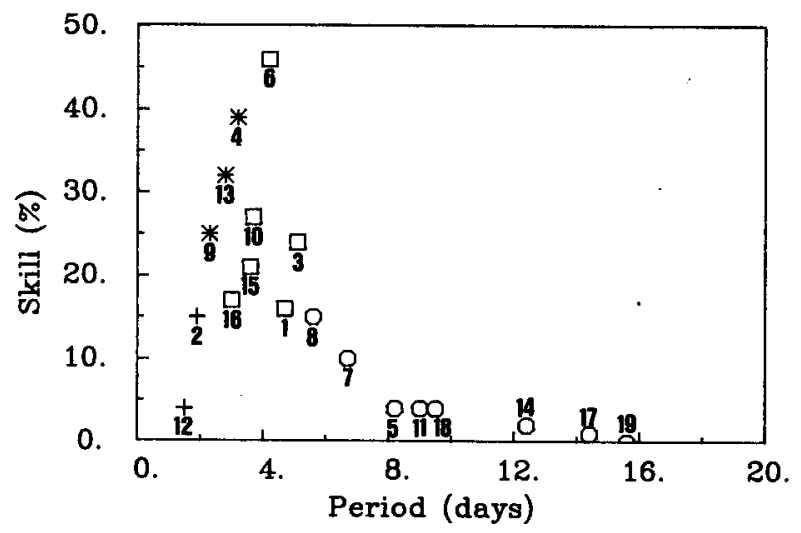

FIG. 7. The skill with which the EOFs in Fig. 6 account for the $\psi$ streamfunction fields of the eigenmodes whose eigenvalues are plotted in Fig. 4b. The eigenmode index of each mode is also plotted; symbols as in Fig. 4b. 

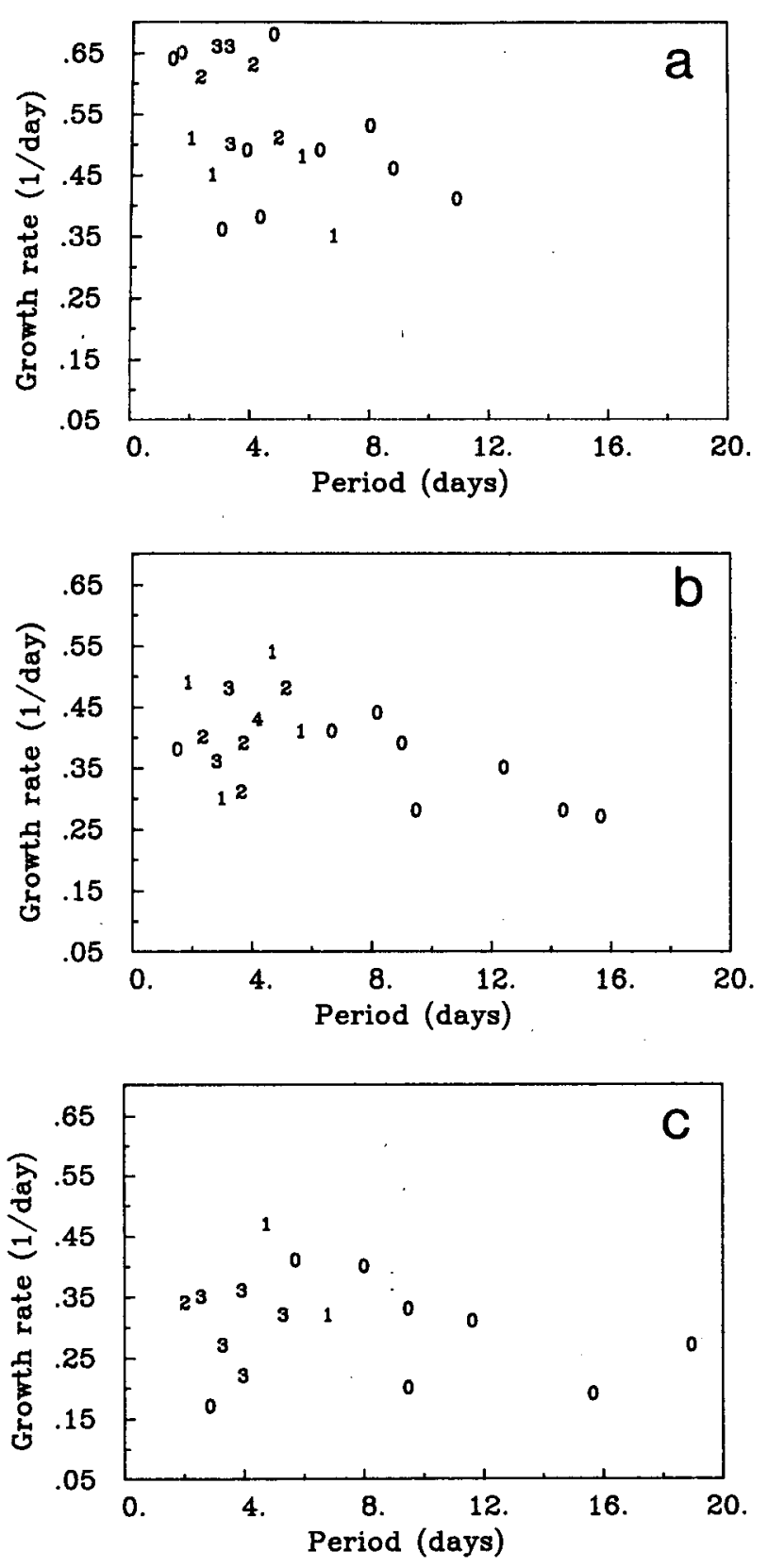

FIG. 8. The result of changing $\Delta \theta_{0}$ in the stability analysis (winter climate, $\Upsilon 21$ ). (a) $\Delta \theta_{0}=24 \mathrm{~K}$, (b) $\Delta \theta_{0}=30 \mathrm{~K}$, and (c) $\Delta \theta_{0}=36$ $K$. Each panel shows the eigenvalues, together with the skill class of $S^{(k)}$ associated with the modes, for the 20 fastest growing modes for each case (modes with periods greater than 20 days are excluded). Key to skill classes: $(0),[0.0,0.1) ;(1),[0.1,0.2) ;(2),[0.2,0.3)$; (3), $[0.3,0.4) ;(4),[0.4,0.5)$.

effect of decreasing the static stability is to increase the growth rates of the modes, the frequencies of the fastestgrowing modes also show a marked increase, so that with $\Delta \theta_{0}=24 \mathrm{~K}$, the cyclogenesis class of mode are now, in general, the fastest growing (though with very large growth rates). However, there are now more modes with very short periods and small spatial scales, which correlate poorly with the leading bandpass fil. tered EOFs. Increasing the static stability so that $\Delta \theta_{1}$ $=36 \mathrm{~K}$ has the opposite effect, making the high-frequency cyclogenesis modes rarer among the fastestgrowing modes, which are now rather slow growing. Fewer very high frequency modes are present, so that highly localized Pacific modes are less common. For the purposes of producing cyclogenesis modes which can be best identified with the observations, and in order to obtain growth rates comparable with multileve: model results (Simons and Hoskins 1976), a static sta.bility parameter corresponding to about $\Delta \theta_{0}=30 \mathrm{~K}$ (Fig. 8b) is required.

\section{d. Surface friction}

In the above it has been assumed that the effect of dissipation, and in particular surface friction, on the perturbation is negligible. It can be shown that the effest of uniform linear dissipation in the $B$-model is simply to shift the modal growth rates by an amount equal to the damping time scale, while leaving the frequencies and structures of the modes unchanged. Thus uniforrn Rayleigh drag and Newtonian cooling with 10 day damping time scales would result only in a decrease of 0.1 day $^{-1}$ in the growth rates, so that we do not expest surface friction to radically change the results. The stability analysis was repeated with Ekman damping irlcluded (damping time scale 7 days), as described in section 2. Figure 9 shows the eigenvalues of the 20 fastest-growing modes together with approximate values of $S^{(k)}$. The growth rates are only about 0.1 day ${ }^{-1}$. less than in the frictionless case (Fig. 8b), so that including an Ekman layer does not drastically damp the fastest-growing modes. Furthermore, the spectrum of modal structures, as indicated by the $S^{(k)}$ values, is also not essentially different from the frictionless casi:. In the following, we will restrict our discussion to the frictionless case.

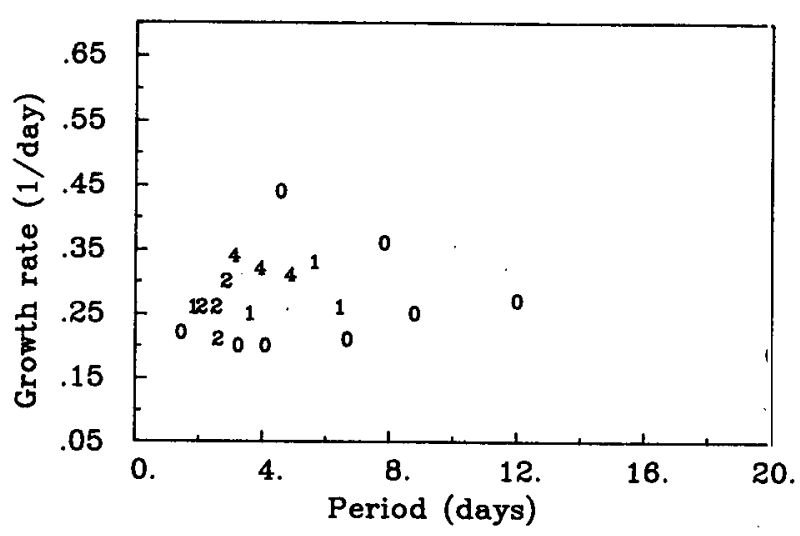

FIG. 9. Effect of Ekman pumping with 7-day damping time scale. Otherwise as in Fig. 8b. 


\section{e. Spatial scale separation}

From the point of view of low-order models, it would be useful to know if the basic-state planetary waves alone contain enough information for the stability analysis. We have thus truncated the six-winter basic state rhomboidally at zonal wavenumber $4(\mathrm{R} 4)$, and also restricted the perturbation to the synoptic-scale regime corresponding to the subset $T 21$ minus $R 4$. The effect of excluding the synoptic scales from the basic state is to weaken the Pacific trough and downstream ridge a little. The effect is more marked in terms of the thermal wind whose maximum over Japan is reduced to only $22.3 \mathrm{~m} \mathrm{~s}^{-1}$, while that over the east coast of North America is strengthened slightly $\left(17.0 \mathrm{~m} \mathrm{~s}^{-1}\right)$, so that the strengths of the two maxima become more comparable.

With the perturbation restricted to $\mathrm{T} 21-\mathrm{R} 4$, there are 80 fewer degrees of freedom and many of the lowerfrequency modes, which are generally planetary-scale, no longer appear. The eigenvalues of the 20 fastest growing modes together with their skill classes are illustrated in Fig. 10. In comparison with Fig. 8b, there is more differentiation in growth rate between the modes, fewer very high-frequency modes, and a tendency for the fastest-growing modes to be cyclogenesis modes with the highest skills. The modal structures (not shown) are rather similar to their T21-basic-state counterparts, and there is little tendency for cyclonic activity to be relatively stronger over the Atlantic, though the very high frequency, highly localized Pacific modes are now largely absent. The above experiment was repeated with the R4 components retained in the perturbation, with indistinguishable results for the modes with periods less than about $4^{1 / 2}$ days; i.e., the cyclogenesis modes.

\section{f. Random phase ensemble averages (RPEAs)}

For the purpose of a convenient comparison of the spatial distribution of the temporal variance that is as-

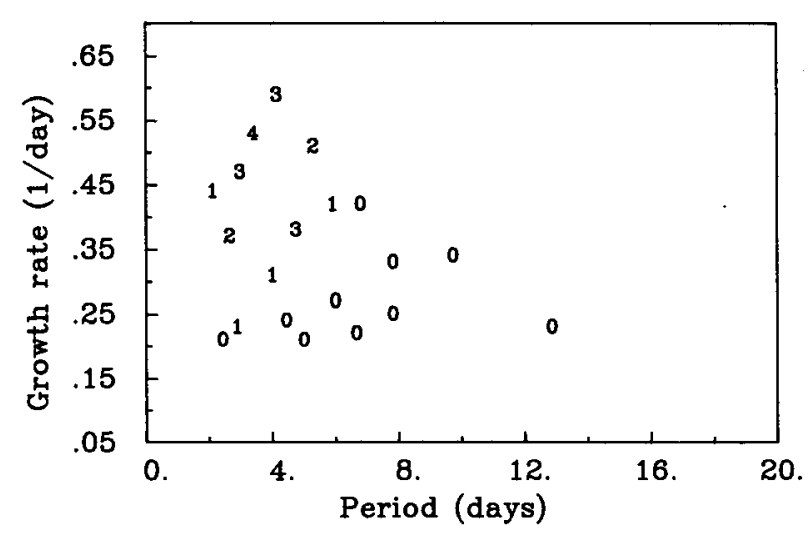

FIG. 10. Effect of spatial scale separation: basic state R4, perturbation T21 - R4. Otherwise as Fig. 8b.

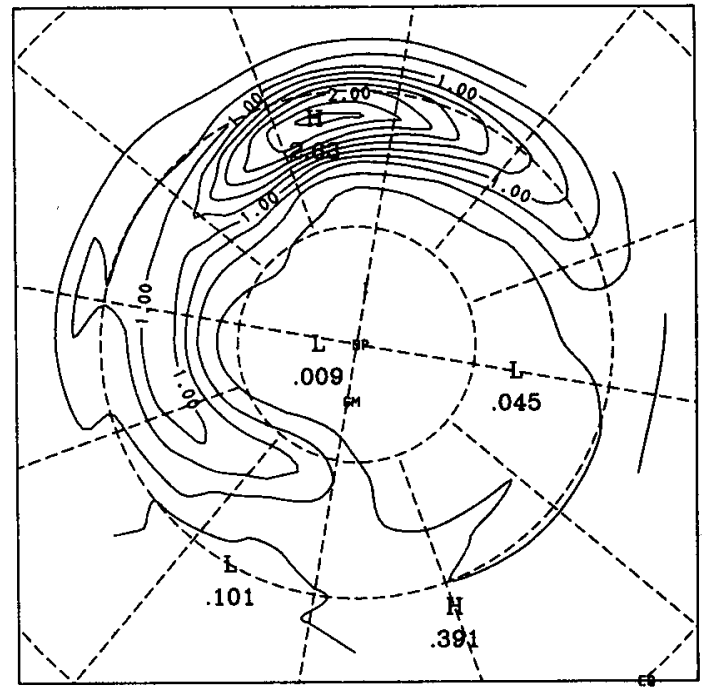

FIG. 11. Composite of the RPEA standard deviations of the six $\psi$-streamfunction modes in Fig. 5. Magnitudes arbitrary.

sociated with a particular normal mode, we adopt the RPEA method of Frederiksen (1982). The ensemble, over which we average, is taken to consist of all phases of the eigenmode with equal probability. Writing the zonal asymmetric part of the real eigenmode $\psi$ streamfunction structure, with arbitrary phase $\alpha$ as

$$
\psi^{\alpha}(\lambda, \mu)=\hat{\psi} e^{i \alpha}+\hat{\psi}^{*} e^{-i \alpha}
$$

where

$$
\hat{\psi}^{\prime}=\sum_{m} \sum_{n} \psi_{n}^{m} e^{i m \lambda} P_{n}^{m}(\mu) .
$$

Then the RPEA variance is defined as

$$
\left\langle\left(\psi^{\alpha}\right)^{2}\right\rangle \equiv \frac{1}{2 \pi} \int_{0}^{2 \pi}\left(\psi^{\alpha}\right)^{2} d \alpha=2|\hat{\psi}|^{2}
$$

Figure 11 shows the result of calculating the square root of the RPEA variance for each of the $\psi$-streamfunction modes in Fig. 5, and then averaging the six envelopes together, giving each mode equal weight. Although the amplitude of the resulting composite or "linear storm track" is arbitrary, we see that taking the leading cyclogenesis modes together captures the observed Pacific storm track (Fig. 3b) quite well, except that it is situated somewhat too far southeastward. However, as we saw from the individual modes in the composite, the Atlantic storm track is too weak relative to that over the Pacific, and has no separate maximum. The resulting linear storm tracks are not found to be very sensitive to the particular selection of the modes in the composite, though selecting modes with shorter periods leads to a stronger, more highly localized Pacific storm track, at the expense of the Atlantic track, and vice versa. 


\section{Instability of anomalous basic flows}

\section{a. The basic flows}

The anomalous basic states considered in this section consist of the six-winter climatology of section 4 to which certain observed low-frequency anomalies have been added. We use a rotated principal component analysis (RPCA) of the low-pass filtered six-winter dataset to obtain the anomalies. Horel ( 1981 ) and Barnston and Livezey (1987) have demonstrated the ability of RPCA to yield robust patterns similar to the teleconnection patterns of Wallace and Gutzler (1981), and the persistent anomaly composites of Dole (1986).

The low-pass filtered (Blackmon and Lau 1980; oscillations with periods greater than approximately 10 days retained ) daily streamfunctions from our six-winter dataset $(6 \times 92$ days $)$ were used in the analysis. The mean seasonal cycle is removed but interannual variability is retained. The spectral fields of the streamfunctions $\psi$ and $\tau$ (as defined in section 2) were transformed to an approximately equal-area grid similar to that used by Barnston and Livezey (1987). The grid extends from $20^{\circ}-85^{\circ} \mathrm{N}$ with a $5^{\circ}$ spacing in latitude. There are 36 points per latitude circle from $20^{\circ}$ to $55^{\circ} \mathrm{N}, 24$ points at $60^{\circ}$ and $65^{\circ} \mathrm{N}, 18$ points at $70^{\circ}$ and $75^{\circ} \mathrm{N}$, and 9 at $80^{\circ}$ and $85^{\circ} \mathrm{N}$. The daily data vector $\mathbf{x}(t)$, from which the covariance matrix was constructed, was made up of the grid point values for $\psi$ and $\tau$ together. The resulting normalized EOFs were scaled by the square root of the corresponding eigenvalue, and the first 20 rotated using an IMSL Varimax library routine. This cutoff point accounts for $86 \%$ of the total temporal variance, and was found to yield patterns most resembling those of Dole (1986) and Barnston and Livezey (1987).

Two of the resulting rotated patterns were selected for their likeness to the composite $500 \mathrm{mb}$ height persistent anomalies of Dole (1986). Rotated EOF 2 (Figs. $12 \mathrm{a}, \mathrm{b})$ accounts for $6.5 \%$ of the low-pass filtered temporal variance, and has a Pacific/North American (PNA)-like structure, which is similar to Dole's Pacific positive composite. Figure $14 a$, b illustrates rotated EOF 13, (3.5\% variance) which is very similar to Dole's Atlantic pattern [Barnston and Livezey's EA pattern ]. In addition, a NAO pattern (rotated EOF $5,5.9 \%$ variance; Figs. $16 \mathrm{a}, \mathrm{b}$ ) was also selected in order to investigate the effect of low-frequency variability in the Atlantic jet exit region. All three patterns have equivalent barotropic structures and are quite robust. Very similar patterns were obtained using 11 winters of German Weather Service data (1967/68-1977/78), and also by an oblique rotation, as well as when only 10 EOFs are rotated. Thus although six winters is an undesirably short period for a low-pass filtered EOF analysis, and the patterns may not be statistically significant, their robustness, as well as their similarity with patterns found in other studies, is evidence of their physical relevance.
Six anomalous basic states are obtained by adding each of the above rotated EOFs (scaling to an ampl:tude of two standard deviations), with a positive and a negative sign to the climatological basic flow. They are illustrated in panels (c) (positive phase anomalies), and (d) (negative phase anomalies) of Figs. 12, 14 and 16 respectively. In each panel, the $\psi$-component of the basic flow is illustrated together with the 15 and $25 \mathrm{mn}$ $\mathrm{s}^{-1}$ isotachs of the associated thermal wind between 850 and $300 \mathrm{mb}$.

\section{b. Linear stability analysis}

The stability analysis was performed using $\Delta \theta_{0}=30$ $K$, and with both the basic state and the perturbation truncated at $T 21$. The eigenvalue of the fastest-growing mode for each of the six cases is given in Table 2. The superposition of low-frequency anomalies generally results in small increases in the growth rates of the fastest-growing modes. As in the previous section, RPEA variances of the leading cyclogenesis mode $\psi$ streamfunctions have been constructed. For each cases, we calculate the square root of the RPEA variance for each of the leading cyclogenesis modes, and average them together to produce a composite or linear storrn track for each anomalous basic flow. Here, the six fasl:est-growing modes with periods between 2 and $4 \frac{1}{2}$ days are taken to define the leading cyclogenesis modes which make up the composites. Thus, the very short:period highly localized Pacific modes are neglectecl, while some of the less regionally confined slower modes with higher Atlantic activity are included. This somewhat accentuates the Atlantic storm track.

\section{1) Pacific/North AMerican CASE (PNA)}

Figure 12 illustrates the PNA case. The main difference between the basic states associated with the positive phase (Fig. 12c; rotated EOF 2 added), and the negative phase (Fig. 12d; EOF subtracted) of the pattern is the anomalous trough/ridge over the east Pacific; though in the negative phase the jet over the east coast

TABLE 2. Eigenvalue of the fastest-growing mode for the six anomalous basic states (section 5 ), and for the winter climatological basi. state (CLIMATE).

\begin{tabular}{lccc}
\hline \hline Experiment & $\begin{array}{c}\text { Angular freq. } \\
\left|\omega_{r}\right| \\
\left({ }^{\circ} \text { day }^{-1}\right)\end{array}$ & $\begin{array}{c}\text { Period, } \\
(\text { days })\end{array}$ & $\begin{array}{c}\text { Growth rate, } \\
\omega_{i} \\
\left(\text { days }^{-1}\right)\end{array}$ \\
\hline CLIMATE & 77 & 4.7 & 0.54 \\
+ PNA & 82 & 4.4 & 0.54 \\
- PNA & 71 & 5.1 & 0.59 \\
+ EA & 85 & 4.2 & 0.63 \\
- EA & 84 & 4.3 & 0.58 \\
+ NAO & 123 & 2.9 & 0.58 \\
- NAO & 88 & 4.1 & 0.64 \\
\hline
\end{tabular}



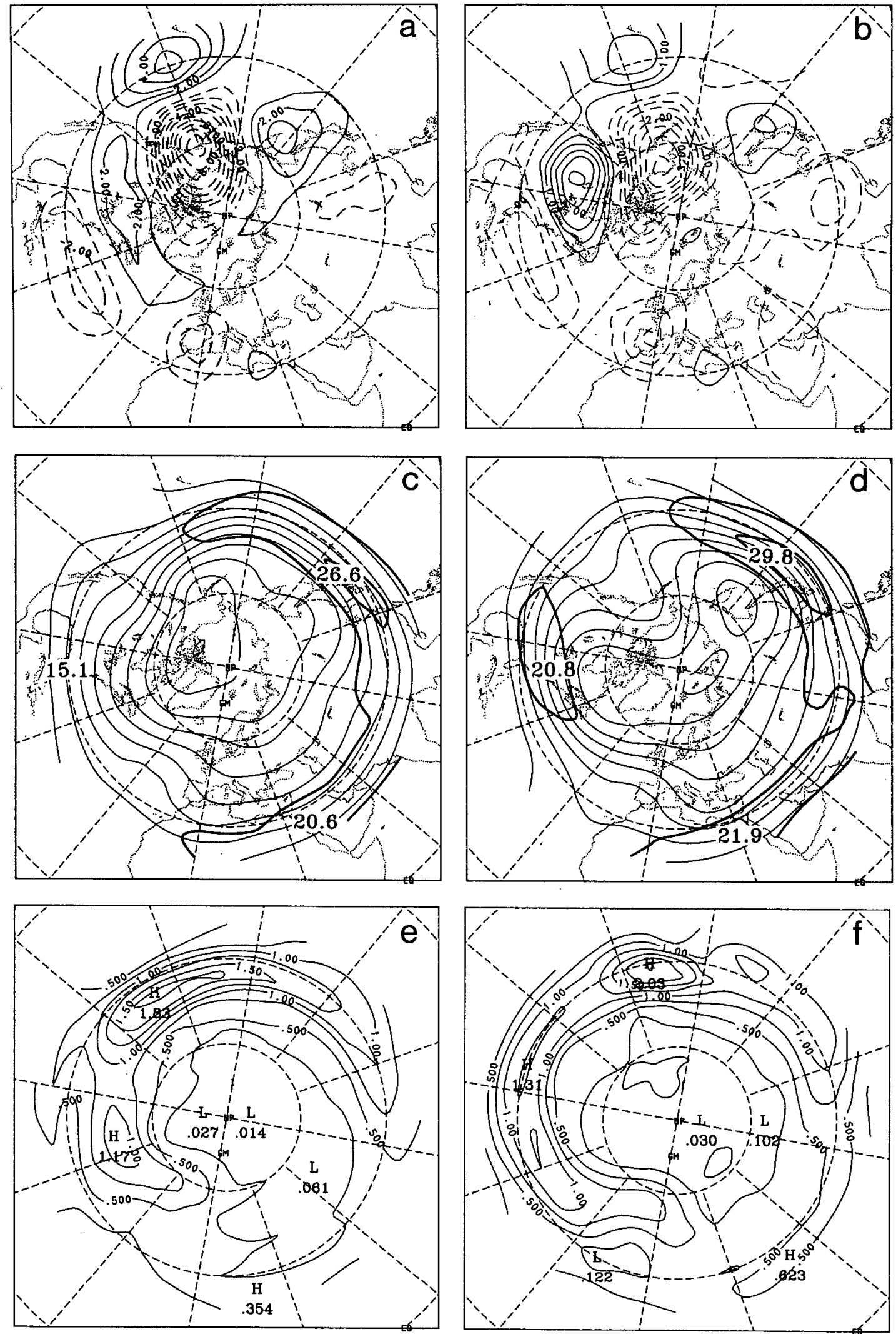

FIG. 12. Stability analysis of PNA basic states. (a) $\psi$-field of rotated EOF 2 (contour interval $10^{6} \mathrm{~m}^{2} \mathrm{~s}^{-1}$ ), (b) corresponding $\tau$-field (contour interval $0.5 \times 10^{6} \mathrm{~m}^{2} \mathrm{~s}^{-1}$ ), (c) and (d) Anomalous basic states obtained by adding/subtracting rotated EOF 2 to/from the winter climatology respectively, details as in Fig. 3a. (e) and (f) Composites of 6 leading cyclogenesis mode RPEAs (see text) for basic states in (c) and (d) respectively, magnitudes arbitrary. 
of North America and the trough over Japan are also stronger and more baroclinic. In both positive and negative phases of the pattern, the associated linear modes (Figs. 12e, f) are strongest over the Pacific, as they were in the climatological case (Fig. 11). In the positive phase (Fig. 12e), the linear modes extend further eastward over the Pacific, consistent with the eastward Pacific jet extension, and there is a weak Atlantic modal maximum in its climatological position. In the negative phase (Fig. 12f), the Pacific modal maximum occurs just upstream of the ridge. Instead of being steered northward around the ridge, the linear "storm track" extends zonally toward North America and then out over the Atlantic. Indeed no individual mode was steered around the ridge. In the negative phase there is scarcely an Atlantic maximum, but the modes propagate northeastward toward northwest Europe. The linear storm tracks are quite robust, and a similar result was obtained using a PNA pattern with a simpler Pacific structure resulting from an oblique rotation.

To get an indication of the extent to which the linear model's sensitivity to the "PNA" anomaly matches atmospheric behavior, episode-composites of bandpass variance have been constructed. First, high amplitude episodes of the rotated principal component (RPC) (i.e., strong projection of the observed data onto the rotated EOF) were identified. Then an rms map of the daily bandpass filtered $\psi$-fields was computed for each episode, and a composite constructed, weighting each episode according to its duration. Episodes of RPC amplitude greater than one standard deviation for longer than a week were included, and composites of both positive and negative sign were constructed. These criteria, although being somewhat weak (the EOF added to the basic state was scaled to an amplitude of two standard deviations), were required to obtain composites of reasonable size. Corresponding composites of the unfiltered $\psi$-field were constructed in parallel. In general, these (not shown) resemble th: basic states in Figs. 12c, d quite closely over the Pacific, although they have a slightly weaker PNA pattern.

Figures $13 \mathrm{a}$ and $13 \mathrm{~b}$ illustrate the rms composites for the positive phase ( 7 episodes), and negative phase ( 3 episodes) of RPC 2 respectively. Although the composites may not be statistically significant, a roug. comparison with the linear modes has been attempted over the Pacific. In the positive phase, the composite Pacific storm track is slightly intensified but little shiftei from its climatological position, in contrast to the eas1ward shift seen in the linear modes. In the negative phase, the Pacific storm track is shifted (if anything) slightly southeastwards, with no tendency for the observed cyclones to be steered northward around the ridge over the northeast Pacific. This suggests that the east Pacific ridge is situated too far north to appreciably effect the Pacific storm track, and that the linear mode behavior may indeed be reasonable.

\section{2) East Atlantic Case (EA)}

Figure 14 illustrates the East Atlantic (EA) cass: Rotated EOF 13 (Figs. 14a, b), has a simple northsouth dipole structure, which produces a ridge in the basic flow over the East Atlantic and western Europe in the positive phase (Fig. 14c), or a trough in the negative phase (Fig. 14d). The positive phase of the EA pattern may be associated with European blocking. The flow in the Pacific jet region is little affected by the superposition of the anomaly.

The associated normal-mode composites are illus-
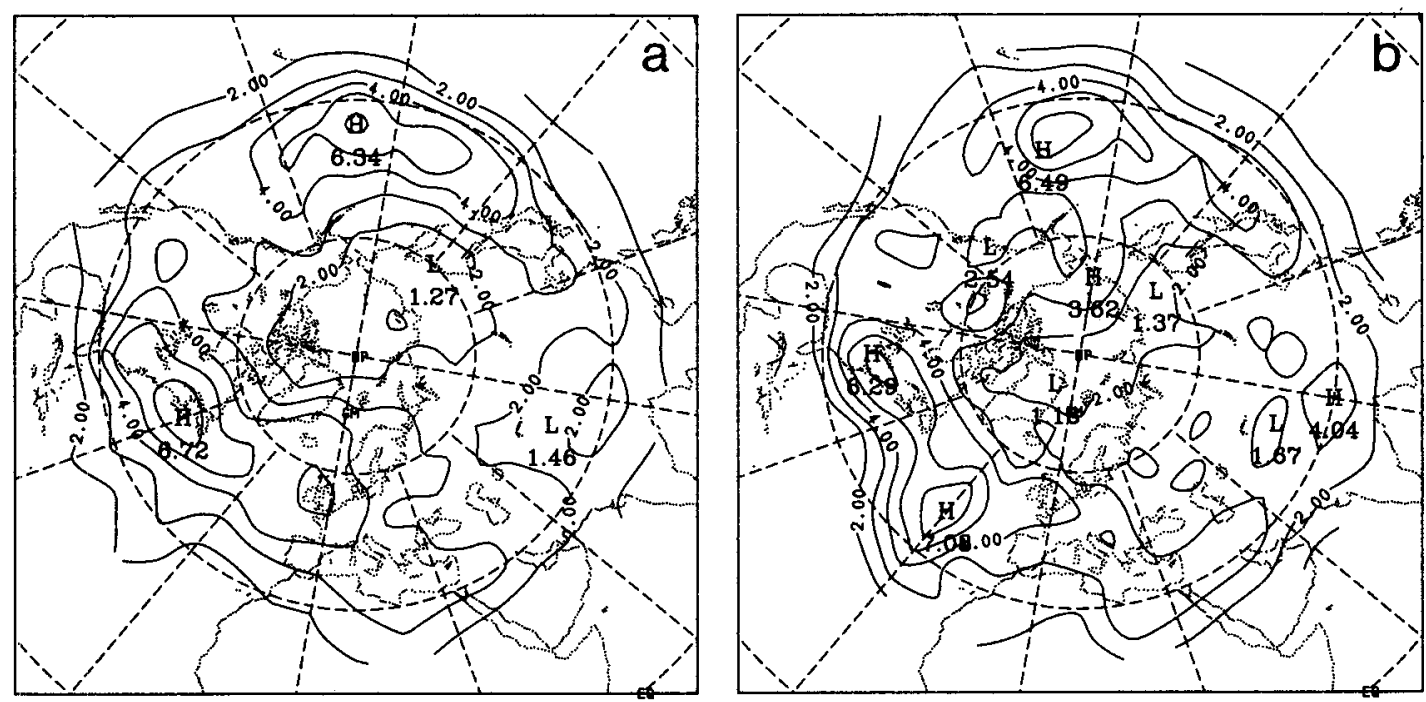

FIG. 13. Composite bandpass rms $\psi$ for episodes of RPC 2 (contour interval $10^{6} \mathrm{~m}^{2} \mathrm{~s}^{-1}$ ). (a) Positive phase ( 7 episodes), (b) negative phase ( 3 episodes). 

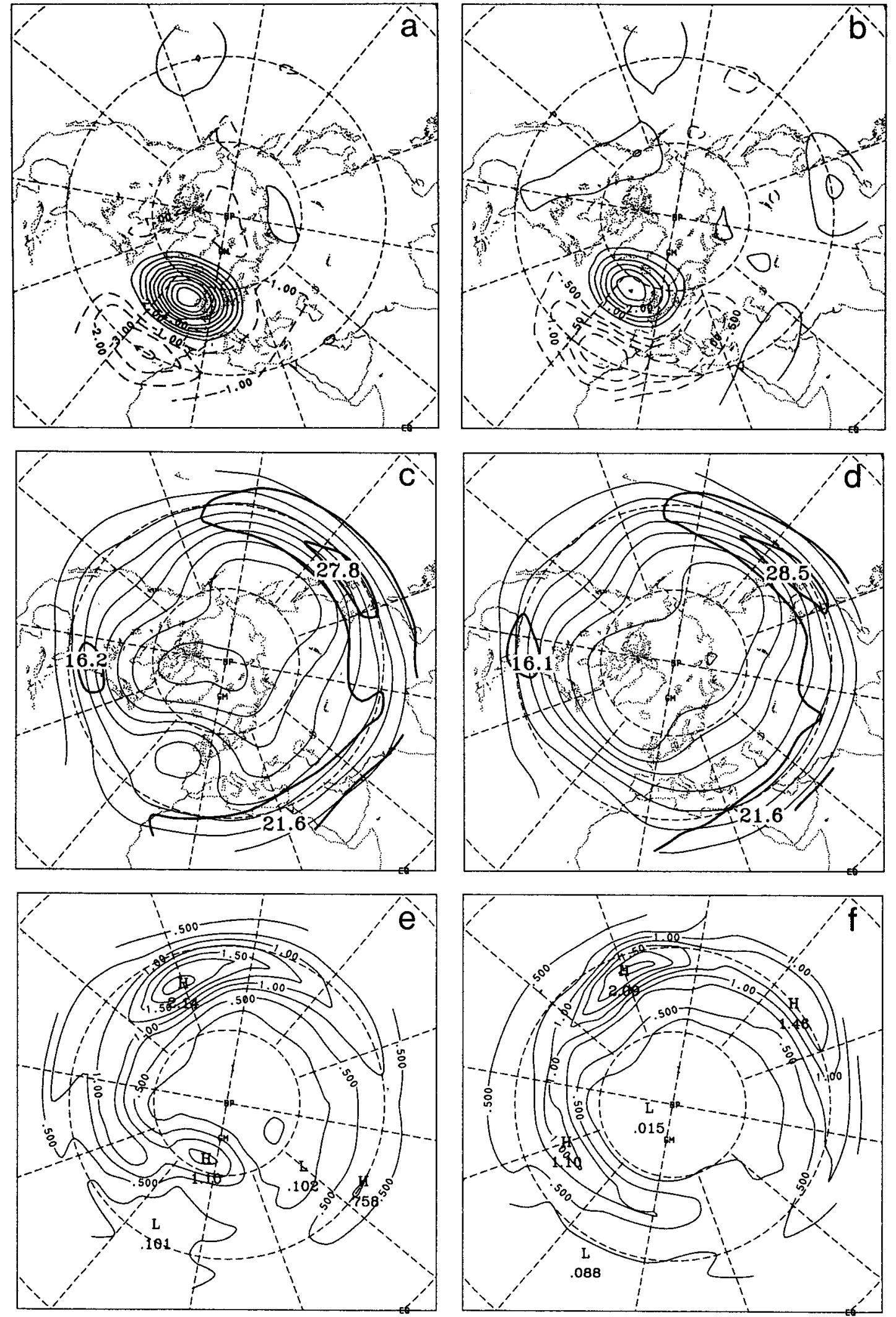

FIG. 14. Stability analysis of EA basic states; as in Fig. 12 but for rotated EOF 13. 
trated in Figs. 14e, f. In this case the main effect of the anomaly is over the Atlantic, where there is an eastward extension and a clear steering of the linear modes by the anomalous ridge/trough over the east Atlantic. Following the same procedure as above, episode-composites of bandpass variance have been constructed for RPC 13. Figure 15a illustrates the composite for the positive phase ( 6 episodes), which indicates that the Atlantic storm track is strongly steered around the anomalous east Atlantic ridge. In the negative phase (Fig. 15b; 3 episodes), the Atlantic storm track extends zonally eastward across the Atlantic. The composites for both phases of the RPC are in good agreement with similar composites constructed by Dole (1986), and indicate that the linear cyclogenesis modes, while being too weak over the Atlantic compared to over the Pacific, are steered by the presence of the EA pattern, in much the same manner as the observed storm tracks.

\section{3) NORTH Atlantic Oscillation CASE (NAO)}

In the cases presented above the Atlantic modal maximum is always much too weak (relative to the Pacific modal maximum), compared with the observed eddy activity. In order to consider the effect of lowfrequency variability in the Atlantic jet region on the Atlantic storm track, we examine here the stability of basic states which include a North Atlantic Oscillation pattern (NAO) (Fig. 16). The anomaly (rotated EOF 5 ) consists of a zonally elongated north-south dipole centered over the northwest Atlantic (Figs. 16a, b), whose principal effect on the flow is to modify the Atlantic jet. In the positive phase (rotated EOF added, Fig. 16c), the Atlantic trough is intensified and extended zonally eastward out over the Atlantic Ocean.
In the negative phase (rotated EOF subtracted, Fig. 16d), the Atlantic trough is weakened while a sharj) southwesterly jet is produced centered over Newfoundland.

The main effect of the anomaly on the leading cyclogenesis modes is as expected over the North AmeIican-Atlantic sector. In the positive phase (Fig. 16e), a clear Atlantic modal maximum is produced, south of the climatological position, which is of comparable magnitude to the Pacific maximum. In the negative phase (Fig. 16f), there is a weak Atlantic maximum, which extends with the anomalous jet, northeastwards toward northwest Europe.

Figure 17 illustrates the episode-composites of bandpass variance for RPC 5 constructed as above. In the positive phase (Fig. 17a, 8 episodes), the Atlantic storm track is somewhat stronger and extends further southeastwards than in the six-winter mean. In the negative phase (Fig. $17 \mathrm{~b}, 5$ episodes), the Atlantic storm track extends northeastwards right over the North Atlantic, with a secondary maximum just weist of Scotland. The linear cyclogenesis modes capture qualitatively the main changes.seen in both the episodecomposites over the Atlantic. The comparatively large amplitude of the linear modes over the Atlantic in the positive phase ( together with their rather southerly position), in contrast to their weakness in the negative phase, suggests that $B$-model's bias towards instability at lower latitudes may be important in this case.

\section{Summary and conclusions}

We have attempted to investigate the role played by persistent anomalies of the large-scale flow in organi:ing linear cyclogenesis, by examining the fastest-grov/-
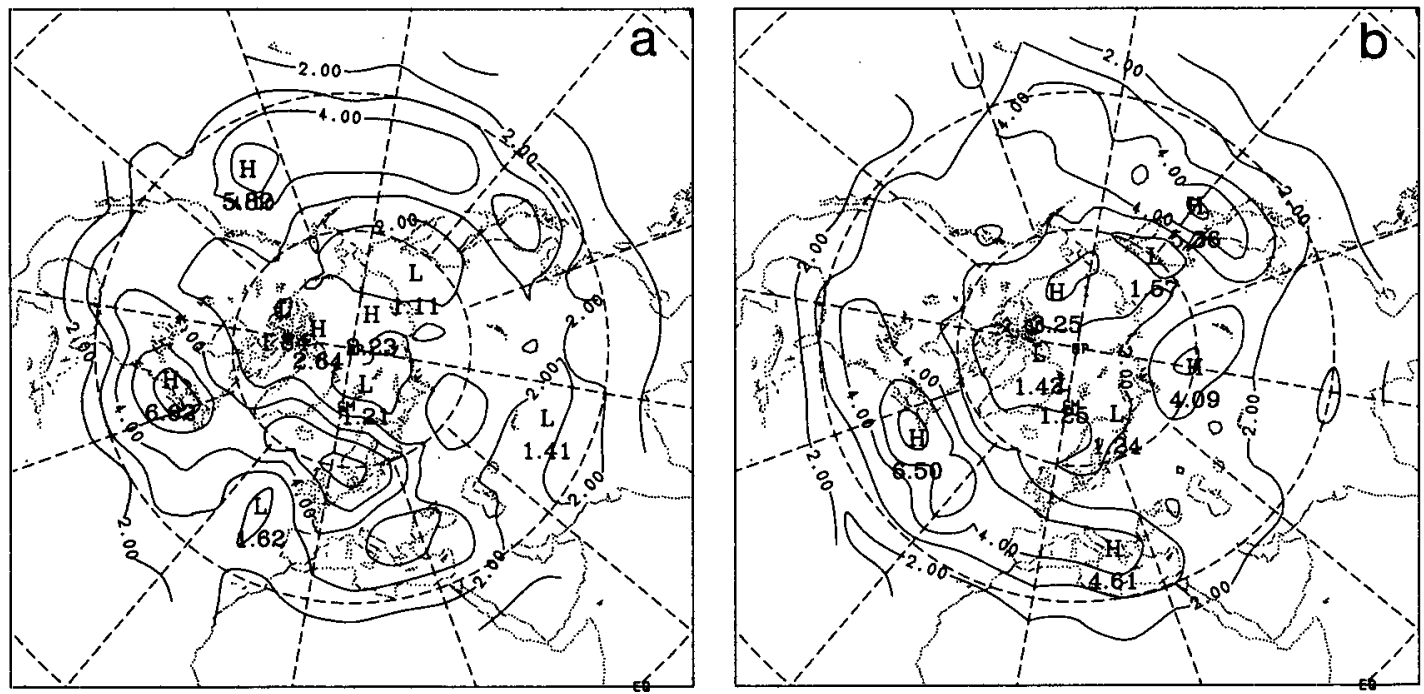

FIG. 15. Composite bandpass rms $\psi$ for episodes of RPC 13 (contour interval $10^{6} \mathrm{~m}^{2} \mathrm{~s}^{-1}$ ).

(a) Positive phase ( 6 episodes), (b) negative phase ( 3 episodes). 

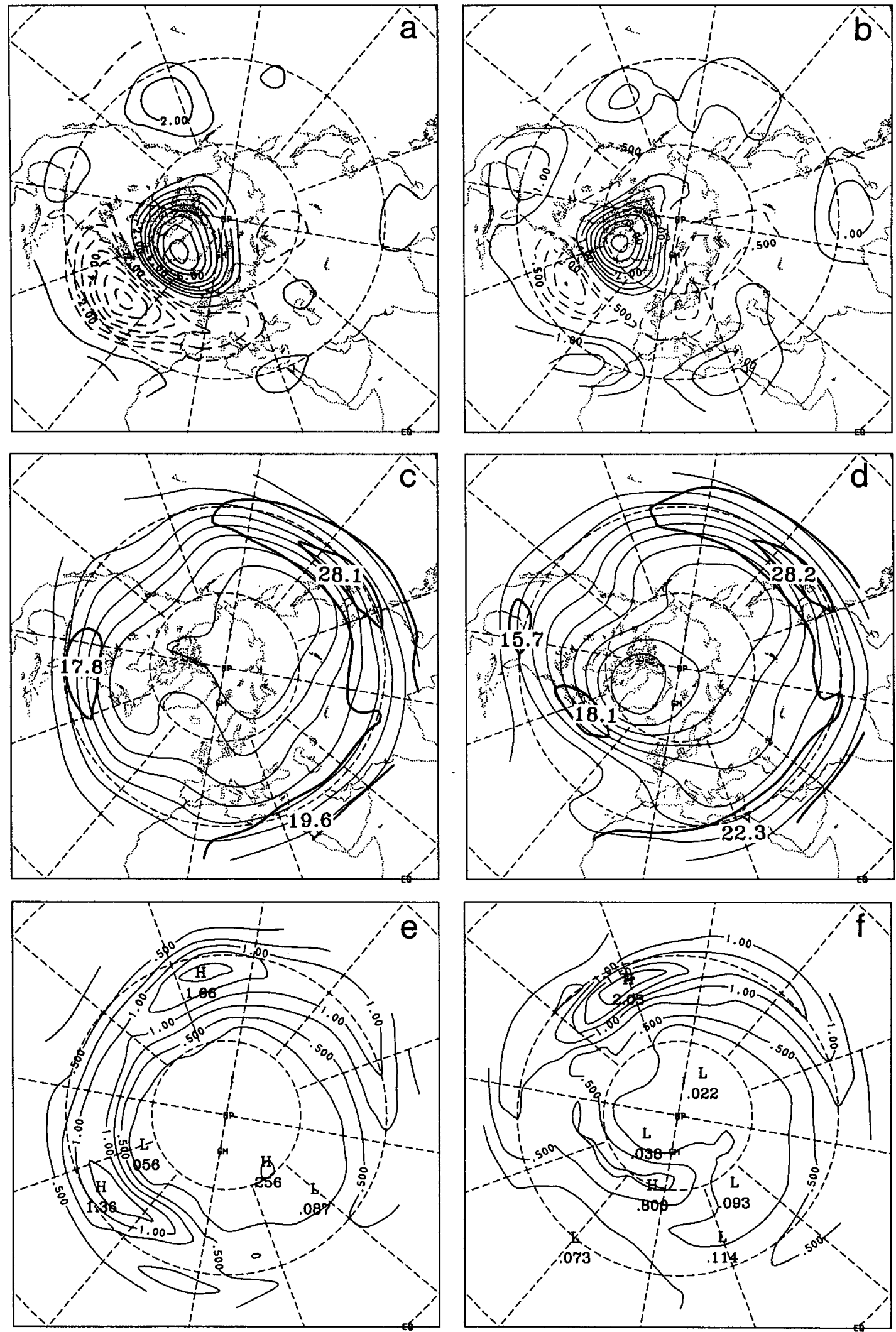

FIG. 16. Stability analysis of NAO basic states; as in Fig. 12 but for rotated EOF 5. 

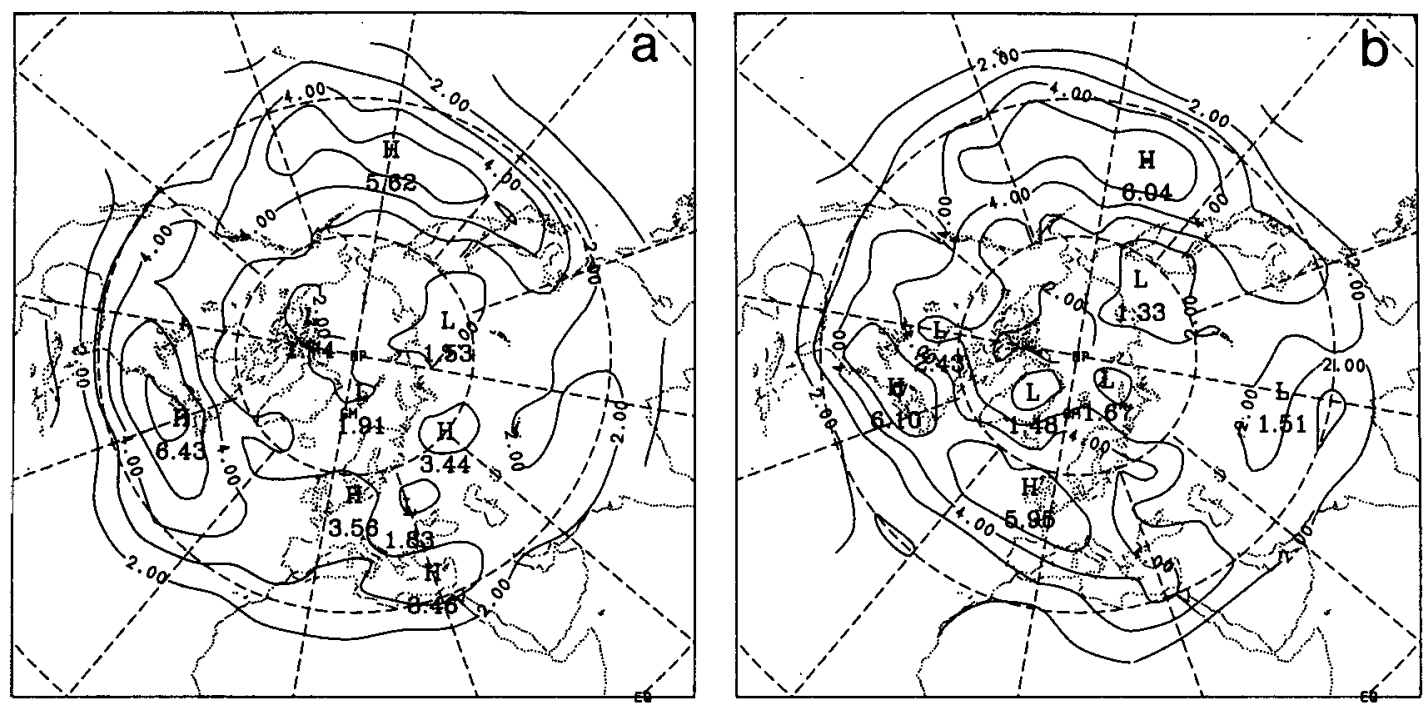

FIG. 17. Composite bandpass rms $\psi$ for episodes of RPC 5 (contour interval $10^{6} \mathrm{~m}^{2} \mathrm{~s}^{-1}$ ).

(a) Positive phase ( 8 episodes), (b) negative phase ( 5 episodes).

ing normal modes of a simple two-level quasi-geostrophic model, linearized about various three-dimensional basic states. The linear stability analysis was first applied to idealized basic states, made up of a climatological zonal-mean flow, to which local Gaussianshaped anomalies were added. It was found, in agreement with Frederiksen (1978b), that the fastest-growing modes with short periods ( $T<4$ days) possess many of the characteristics of observed midlatitude cyclones, being regionally organized, with maximum amplitude slightly downstream of the local baroclinity maximum. The linear instability of a six-winter climatological flow was then examined. By means of a pattern correlation it was shown that the modes with periods of 3-4 days have structures which resemble most closely the observed leading bandpass EOFs. An equal-weight composite (by amplitude) of the six fastest growing modes with periods less than 4 days was then taken as being representative of the variability associated with the leading cyclogenesis modes.

The leading cyclogenesis modes are found to approximate the Pacific storm track reasonably well, but not the Atlantic track, whose strength is seriously underestimated. The linear modes tend to be less regionally confined in longitude than the observed storm tracks, and there is more cyclonic activity over North America than observed. At first sight, the linear result is quite understandable in view of the strong baroclinity of the Pacific jet, and the much weaker Atlantic baroclinity. However, it is not clear that the $B$-model used in this study deals with the Atlantic jet properly. Frederiksen (1982) has shown that a two-layer $(P$-) model with a fully variable Coriolis parameter has quite localized modes in both Atlantic and Pacific regions. Frederiksen and Bell (1987) have found that as more levels are incorporated so that the local shears are better resolved, the storm tracks in the Atlantic, Pacific (and Siberian) regions become more localized.

Introducing Ekman damping is found to only modestly reduce the growth rates of the leading cyclogenesis modes, while leaving their character rather unchanged. The static stability is a tunable parameter in the twolevel model, and is chosen so as to mimic multilevel results. Varying this parameter not only significant.y changes the modal growth rates, but also the prevalence of high-frequency cyclogenesis modes among the fastest-growing modes, as previously found by Frederiksen (1982).

Little change in the basic character of the leadirig cyclogenesis modes resulted from truncating the basic state rhomboidally at wavenumber 4 . Further limitirg the perturbation to the truncation subset T21 minus R4 had no detectable effect on the cyclogenesis modes. Thus, in principle, a linear stability analysis should be applicable to the task of parameterizing the synoptioscale eddies in a planetary wave low-order model, as suggested by Frederiksen (1979a), and examined in a very low-order model by Rheinhold and Pierrehumbert (1982).

To shed further light on this possiblity and to examine the effect (on the fastest-growing normal modeis) of including persistent anomalies in the basic state, the linear stability analysis was applied to anomalous flows, produced by superimposing rotated EOFs of low-pass filtered observed data, onto the climatology. The $\mathrm{r}(\mathrm{)}-$ tated EOFs comprised a PNA-like pattern, and EA and NAO north-south dipoles. Adding or subtracting these anomalies to/from the climatic basic state was found to result in significant deflections of the normal-mode wavetrains from their climatological positions, partioularly in the EA and NAO cases. This was less true in the PNA case where the Pacific ridge has a blocking 
effect, but is probably situated too far north for the modes to be steered around the ridge. In both EA and PNA cases, maximum modal activity is again situated over the Pacific, with activity smeared over North America and the Atlantic storm track mostly relatively weak. The NAO pattern is centered over the northwest Atlantic and thus affects the Atlantic jet profoundly, and indeed results in storm-track like cyclogenesis modes of reasonable strength over the Atlantic. However, Atlantic activity is never stronger than that over the Pacific, and it seems unlikely that low-frequency variability in the Atlantic jet region can account for the failure of the climatological stability analysis over the Atlantic.

To get an idea of the degree to which the linear model's sensitivity to the presence of persistent anomalies matches atmospheric behavior, composite maps of bandpass variance during high-amplitude episodes of the RPCs were constructed. These maps suggest that the linear modes reflect qualitatively the main local changes occurring as a result of the persistent anomalies. In the NAO case, the composite maps reinforce the suspicion that the bias toward low latitudes inherent in the $B$-model's instability characteristics is an important factor in the models general failure to capture the Atlantic storm track.

From the standpoint of the simple linear $B$-model, Pacific cyclogenesis (and cyclonic activity) appears to be dominated by the regionally strong climatological stationary waves, with low-frequency variability modulating the modes. In contrast, the Atlantic modal activity shows more sensitivity to the presence of persistent anomalies, and is weakly determined by the stationary waves. However, the B-model is probably not adequate for use as part of a synoptic-eddy parameterization for a low-order climate model. Nonetheless, the model's cyclogenesis-type normal modes do exhibit sensitivity to the presence of persistent anomalies, which show encouraging similarities with atmospheric data. Thus, the use of a slightly more sophisticated twolevel model (the $P$-model), might well be very promising regarding a parameterization.

Acknowledgments. We wish to thank J. Egger, and $J$. Frederiksen for helpful discussions and correspondence respectively, and an anonymous reviewer for suggesting the bandpass eddy composites in section 5 . This research was supported by the German Federal Ministry of Research and Technology.

\section{REFERENCES}

Barnston, A. G., and R. E. Livezey, 1987: Classification, seasonality and persistence of low-frequency atmospheric circulation patterns. Mon. Wea. Rev., 115, 1083-1126.

Blackmon, M. L., 1976: A climatological spectral study of the 500 mb geopotential height of the Northern Hemisphere. J. Atmos. Sci., 33, 1607-1623.

_- and N.-C. Lau, 1980: Regional characteristics of the Northern
Hemisphere wintertime circulation: A comparison of the simulation of a GFDL general circulation model with observations. J. Atmos. Sci., 37, 497-514.

- and G. H. White, 1982: Zonal wavenumber characteristics of Northern Hemisphere transient eddies. J. Atmos. Sci., 39, 19851997.

Dole, R. M., 1986: Persistent anomalies of the extratropical Northern Hemisphere wintertime circulation: structure. Mon. Wea. Rev., 114, 178-207.

Egger, J., and H.-D. Schilling, 1983: On the theory of long-term variability of the atmosphere. J. Atmos. Sci., 40, 1073-1085.

Atmos. Sci., 41, 779-788.

Elsaesser, H. W., 1966: Evaluation of spectral versus grid methods of hemispheric numerical weather prediction. J. Appl. Meteor., $5,246-262$.

Frederiksen, J. S., 1978a: Growth rates and phase speeds of baroclinic waves in multi-level models on a sphere. J. Atmos. Sci., 35, 1816-1826.

- 1978b: Instability of planetary waves and zonal flows in twolayer models on a sphere. Quart. J. Roy. Meteor. Soc., 104, 841 872.

- 1979a: The effects of long planetary waves on the regions of cyclogenesis: Linear theory. J. Atmos. Sci., 36, 195-204.

_ 1979b: Baroclinic Instability of zonal flows and planetary waves in multilevel models on a sphere. J. Atmos. Sci., 36, 23202335.

- 1982: A unified three-dimensional theory of the onset of blocking and cyclogenesis. J. Atmos. Sci., 39, 969-982.

— baroclinic, barotropic and topographic instability. J. Atmos. Sci., 44, 2200-2218.

Horel, J. D., 1981: A rotated principle component analysis of the interannual variability of the Northern Hemisphere $500 \mathrm{mb}$ height field. Mon. Wea. Rev., 109, 2080-2092.

Hoskins, B. J., M. E. McIntyre and A. W. Robertson, 1985: On the use and significance of isentropic vorticity maps. Quart. J. Roy. Meteor. Soc., 111, 877-946.

Illari, L., and J. C. Marshall, 1983: On the interpretation of eddy fluxes during a blocking period. J. Atmos. Sci., 40, 2232-2242.

Lorenz, E. N., 1960: Energy and numerical weather prediction. Tellus, 4, 364-373.

Metz, W., 1986: Transient cyclone-scale vorticity forcing of blocking highs. J. Atmos. Sci., 43, 1467-1483.

- 1987: Transient eddy forcing of low-frequency atmospheric variability. J. Atmos. Sci., 44, 2429-2439.

Phillips, N. A., 1954: Energy transformations and meridional circulations associated with simple baroclinic waves in a two-level, quasi-geostrophic model. Tellus, 6, 273-268.

Pierrehumbert, R. T., 1984: Local and global baroclinic instability of zonally varying flow. J. Atmos. Sci., 41, 2141-2162.

, 1986: The effects of local baroclinic instability on zonal inhomogeneities of vorticity and temperature. Adv. Geophys., 29, 165-182.

Rheinhold, B. B., and R. T. Pierrehumbert, 1982: Dynamics of weather regimes: Quasi-stationary waves and blocking. Mon. Wea. Rev., 110, 1105-1145.

Simmons, A. J., and B. J. Hoskins, 1976: Baroclinic instability on the sphere: Normal modes of the primitive and quasi-geostrophic equations. J. Atmos. Sci., 33, 1454-1477.

$\longrightarrow$, and - 1978: The lifecycles of some nonlinear baroclinic waves. J. Atmos. Sci., 35, 414-432.

Shutts, G. J., 1986: A case study of eddy forcing during an Atlantic blocking event. Adv. Geophys., 29, 135-162.

Vautard, R., and B. Legras, 1988: On the source of midlatitude lowfrequency variability. Part II: Nonlinear equilibration of weather regimes. J. Atmos. Sci., 45, 2845-2867.

Wallace, J. M., and D. S. Gutzler, 1981: Teleconnections in the geopotential height field during the northern hemisphere winter. Mon. Wea. Rev., 109, 784-8'2. 